\title{
Recent advances in wearable optical sensors automation pow- ered by battery versus skin-like battery-free devices for per- sonal healthcare - A review
}

\author{
Nikolay L Kazanskiy ${ }^{1,2}$, Muhammad A Butt ${ }^{1,3^{*}}$, Svetlana N Khonina ${ }^{1,2}$, \\ 1 Samara National Research University, 443086 Samara, Russia; butt.m@ssau.ru (M.A.B). \\ 2 IPSI RAS-Branch of the FSRC “Crystallography and Photonics” RAS, 443001 Samara, Russia; kazan- \\ skiy@ipsiras.ru (N.L. K); khonina@ipsiras.ru (S.N.K). \\ 3 Warsaw University of Technology, Institute of Microelectronics and Optoelectronics, Koszykowa 75, 00-662 \\ 5, Warszawa, Poland \\ * Correspondence: Muhammad A Butt; butt.m@ssau.ru
}

\begin{abstract}
Currently, old-style personal medicare techniques rely mostly on traditional methods, such as cumbersome tools and complicated processes, which can be time-consuming and inconvenient in some circumstances. Furthermore, such old methods need the use of heavy equipment, blood draws, and traditional bench-top testing procedures. Invasive ways of acquiring test samples can potentially cause patients discomfort and anguish. Wearable sensors, on the other hand, may be attached to numerous body areas to capture diverse biochemical and physiological characteristics as a developing analytical tool. Physical, chemical, and biological data transferred via the skin is used to monitor health in various circumstances. Wearable sensors can assess the aberrant conditions of the physical or chemical components of the human body in real-time, exposing the body state in time, thanks to unintrusive sampling and high accuracy. Most commercially available wearable gadgets are mechanically hard components attached to bands and worn on the wrist, with form factors ultimately constrained by the size and weight of the batteries required for the power supply. Basic physiological signals comprise a lot of health-related data. The estimation of critical physiological characteristics, such as pulse inconstancy or variability using photoplethysmography (PPG) and oxygen saturation in arterial blood using pulse oximetry, is possible utilizing analysis of the pulsatile component of the bloodstream. Wearable gadgets with "skin-like" qualities are a new type of automation that is only starting to make its way out of research labs and into pre-commercial prototypes. Flexible skin-like sensing devices have accomplished several functionalities previously inaccessible for typical sensing devices due to their deformability, lightness, portability, and flexibility. In this paper, we studied the recent advancement in battery-powered wearable sensors established on optical phenomena and skin-like battery-free sensors which brings a breakthrough in wearable sensing automation.
\end{abstract}

Keywords: Wearable sensors; skin-like, heart rate monitoring; continuous glucose monitoring; battery-free sensors.

\section{Introduction}

Miniaturization advances have led to several wearable sensors that are now being employed in a variety of biomedical applications [1,2]. Some of these have been ingrained in people's daily lives [3]. Smart bands and smart watches with pulse monitors, pulse oximeters, accelerometers, gyroscopes, and other sensors are one example $[4,5,6]$. Implantable devices have been utilized since the 1950s when the first pacemaker was utilized to bring back a regular heart rhythm by continually pumping just the ventricle [7]; since then, implantable automation has evolved to include the most modern implants, for instance Ocular and Cochlear implants [8,9]. Even though biomedical implants are vital to the advancement of medicare, they frequently necessitate the assistance of a trained 
specialist [10]. Wearables, on the other hand, is more intelligible and may be employed deprived of the need for medical or technical competence [11]. Its purpose is to harvest data without requiring any surgical processes or the insertion of materials that are more likely to cause long-term negative effects. These sensors can be worn anywhere you want to wear them. While this sounds like an exaggeration, with sensors implanted in clothing and other form factors besides wrist-worn devices, it is principally true. Figure 1 shows the rise of wearables and future of wearable technology [12].

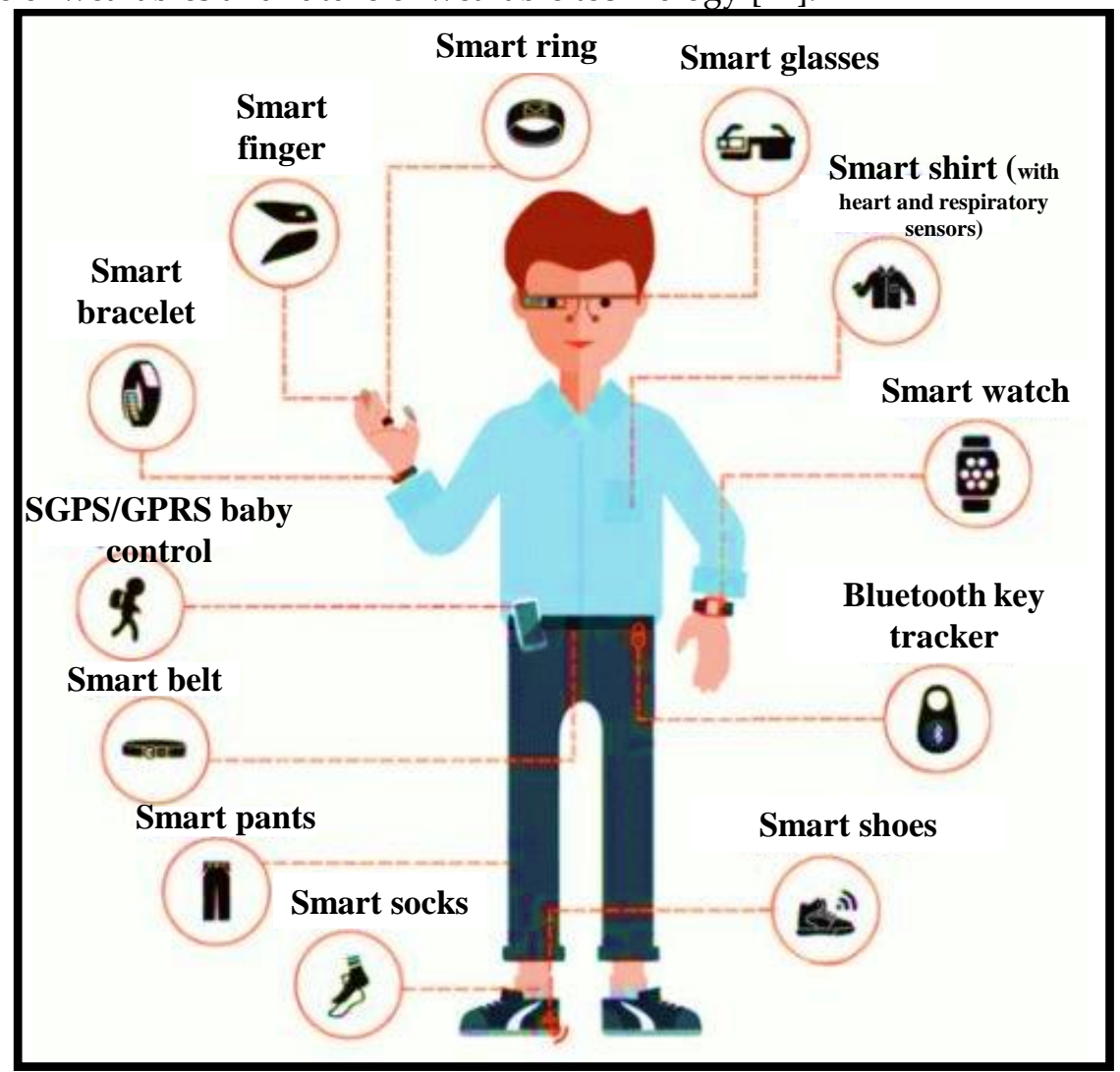

Figure 1. Wearable sensors can be worn almost anywhere on the body for daily activities including health monitoring [12].

The application of wearable devices and arrangements to accomplish immediate recognition of variations in patient status necessitating therapeutic intervention is a rising topic of study in the field of wearables. The care of patients with chronic obstructive pulmonary disease (COPD) is one instance of this sort of wearables use. Early diagnosis of exacerbation events is an important objective in the therapeutic care of patients with COPD. Exacerbations, which are described as bouts of augmented dyspnea, cough, and a variation in the volume and type of sputum, are a regular occurrence in the natural course of COPD, and they can cause functional impairments and disability. Exacerbations should be detected and treated as soon as possible to avoid worsening clinical conditions and the need for emergency department care or hospital admission. Remote monitoring devices can aid in the early detection of patterns in a patient's health state that indicate an impending exacerbation. One way to address the problem of early identification of exacerbation episodes is to monitor variations in a patient's level of activity and presume that a drop in activity level indicates the possibility of a deterioration of the individual's clinical state without further monitoring $[13,14]$.

Atallah et al. have created an ear-worn sensor that may be employed to track activities and degrees of effort in patients with COPD [15]. The researchers were able to recognize numerous different types of physical activities as well as the intensity of those activities using powerful machine learning techniques and a single ear-worn sensor. Steele et al. [16] and Belze et al. [17] measured human movement in 3D over three days and found that the extent of the acceleration vector verified in victims with COPD was correlated 
with measures of victim status like the six-minute walk distance, the FEV1 (Force Expiratory Volume in One Second), the severity of dyspnea, and the physical function domain of the health-related quality of life scale. Hecht et al. [18] proposed an algorithm established on data gathered using a single unit for a minute-by-minute study of victims' activity levels. The method was put to the test in 22 victims for 14 days. The scientists also employed a simple empirically created algorithm to assess whether the person was wearing the gadget, allowing them to keep track of compliance. Another noteworthy finding from the same study was that during the first few days of assessment, individuals tended to increase their activity level. This finding shows that, if monitoring is done regularly, it should be done for long enough to prevent noticing transient regularly, it should be done for long enough to prevent noticing transient effects caused by the fact that the subject is aware that he or she is being watched.

Wearable sensing automation has quickly evolved from a science fantasy concept to a wide range of well-established user and medical devices [19]. The affordability and userfriendly arrangement offered by developments in miniaturized electronics, the development of smartphones and connected devices, an increasing consumer wish for health awareness, and the unmet prerequisite for medical practitioners to uninterruptedly acquire medical quality data from their victims are all contributing to the expansion of wearable sensors [20]. Despite initial success, there is still a thirst for even more data from the body. Most of the sensing modalities including in current wearables (pulse, galvanic skin response, etc.) are non-specific, hence this desire remains unmet (e.g., how many factors can increase your pulse, or trigger you to sweat). Additionally, most wearable sensor devices use techniques that have been around for years. Even the most complex wearables, such as continuous transdermal glucose monitors, benefit from over 30 years of advancements in enzyme electrodes discovered in basic and ultra-low-cost finger-prick glucose test strips. Transdermal glucose assessment is, in fact, possibly the only widely employed wearable sensor that continuously monitors the state of a serious condition (diabetes) [21, 22].

Figure 2 is a conceptual picture of a remote surveillance system [19]. Wearable sensors collect physiological and movement data, allowing victims' status to be monitored. Sensors are employed in different ways depending on the therapeutic use of interest. When monitoring victims with congestive heart failure or COPD who are receiving the clinical intervention, sensors to monitor vital signs would be deployed. Sensors for recording movement data might be employed in applications like monitoring the efficacy of home-based recovery programs in stroke survivors or the usage of mobility assistance equipment in the elderly. Victims' data is transmitted to a mobile phone or an access point through wireless transmission, which is then relayed to a distant center over the internet. Data processing applied throughout the arrangement detects emergency circumstances and an alert message is delivered to a trauma service center to give rapid aid to victims. Family members and caregivers are contacted in the event of an emergency, but they may also be informed in other instances, such as when the victim needs help taking his/her prescriptions. Clinical staff can remotely examine a victim's condition and be notified if a medical decision must be taken. 


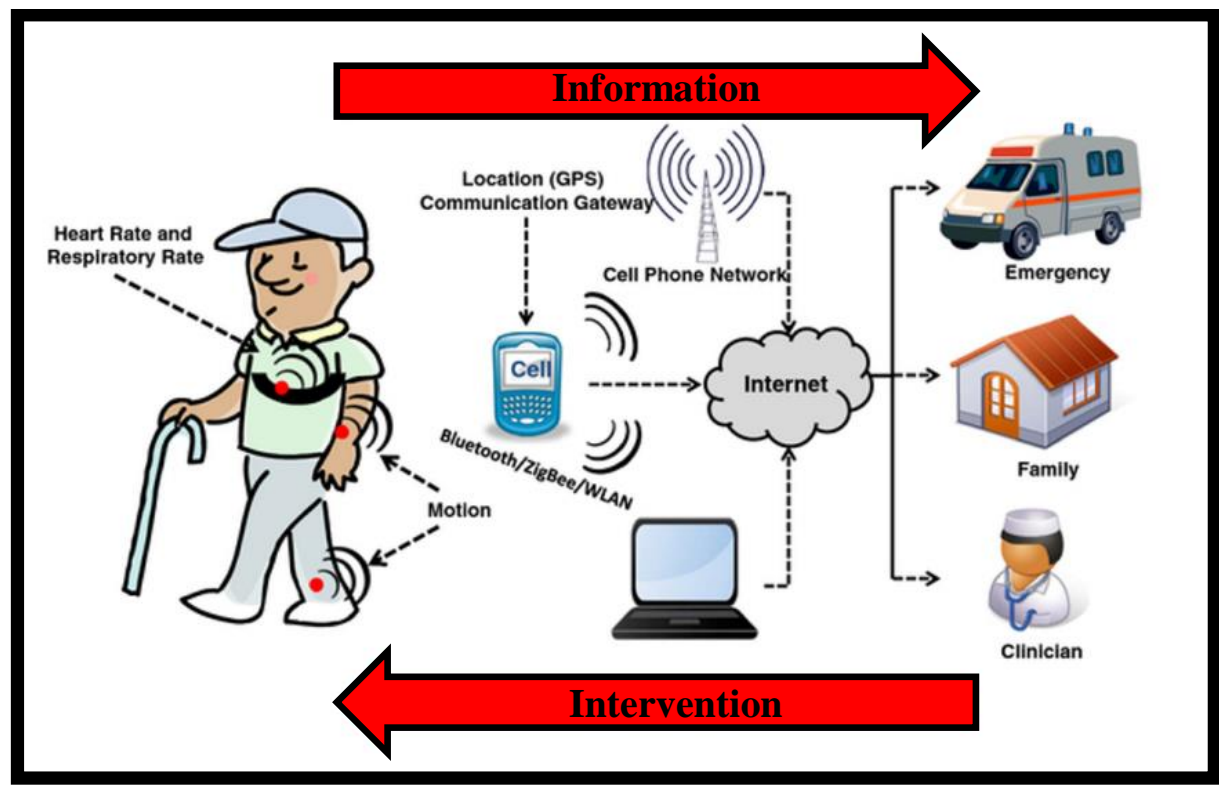

Figure 2. Design of a remote health observing arrangement established on wearables. Health-related data is collected via body-worn wireless devices and transferred to the caregiver via a data gateway for instance a mobile phone. Caregivers can utilize this data to execute interventions as required [19].

Almost every analyte that a clinician could want to assess from a victim can now be measured using diagnostic instruments [23, 24, 25]. Unfortunately, such devices are not wearable, and blood draws and traditional bench-top analysis procedures are still required. So, the key issue on many people's minds is: how can wearable sensor automation begin to cross over into modalities that detect more precise physiological issues, such as validating a child's health while in the mother's womb by tracking mechanical fetal motion? Or tell the difference between a deadly seizure and greater physical exertion? Or a warning that an athlete or worker is becoming severely dehydrated? Or informing the health-conscious about how much overly refined white bread boosted their blood sugar levels? Or mapping and controlling viral infection in a community before most of the population gets symptomatic?

This review is devoted to the recent advancements in wearable sensors established on optical phenomena. We tried our best to review two types of wearable sensors: 1) optical sensors powered by battery, 2) battery-free skin-like sensors. The paper is planned in the following manner. In section 2, the global market and demand for wearable optical sensors are discussed which gives an insight into the importance of this topic. In section 3 , a brief description of the working mechanism of the unintrusive wearable optical sensors is presented. It has become quite simple to receive the body data by placing a compact sensing device on the body. In section 4, the continuous body surveillance devices are considered which are powered by the battery. This section has two sub-parts. In sub-section 4.1, the recent developments and several types of commercially available pulse surveillance devices are reviewed. The place of wearing those devices on the human body plays a significant role in collecting precise data. In sub-section 4.2 , the recent advancements in continuous glucose surveillance devices are reviewed. Afterwards, the new topic related to skin-like wearable sensors is recently emerging which is a talk of the town is discussed in section 5. These sensors are highly attractive, flexile, portable and do not require an integrated battery. The paper ends with final remarks in section 6.

\section{Demand for wearable optical sensors}

Even though both electronics and photonics are important in the prospect of wearables, this paper will concentrate on wearable optical devices. Optical sensors are expected to account for $13 \%$ of the wearable market by 2020 , with optical and optoelectronic (OE) technologies also playing a role in other market segments for instance chemical or elastic 
and pressure sensors. In the recent years, several platforms have been used to produce optical sensors for refractive index sensing applications [26, 27, 28, 29, 30]. Optical sensors are distinct in that they are resistant to electromagnetic radiation, can probe nanoscale volumes, permit unintrusive examination of biological substance at comparatively deep penetration depths, and frequently use low-cost, water and corrosion resistant sensing components. These resources have been used to detect and quantify ion, protein, and viral conc., as well as pulse, blood pressure (BP), blood oxygenation, abdominal and thoracic respiration rate, targeted localized bending, and movement. Optical sensors, like all other sensing devices, must handle the magnified problems of proper signal-to-noise ratio (SNR), restricted dynamic range, signal specificity, and user variability in the setting of wearable devices. Furthermore, there is the issue of surrounding light interfering with signal readings, as well as poor light penetration into the skin and other bio-fluids, which is unique to optical sensing devices. New optical sensing elements and integration techniques, such as photonic textiles [31], innovative colourimetric [32] and fluorometric materials [33], and flexile photonics [34] are currently being researched to solve these difficulties.

By 2025, the worldwide wearable sensing industry is expected to be worth $\$ 5.5$ billion, with currently developing technologies accounting for nearly a third of that total [35]. Over 1/10 of Americans now possess a wearable sensing gadget, such as a specialized fitness surveillance device, a threefold increase from 2012 [36]. Fitness trackers and smartwatches can create personalized health profiles by gathering data on the pulse, blood oxygen level, movement, speed, step count, and even eating and sleeping patterns using mobile phones and cloud connections [37]. Such gadgets are especially appealing for athome health surveillance, particularly for the rising number of seniors who are living independently. Wearables can offer a reliable and thorough victim health record, minimize the resource load on hospitals, and expedite the reaction time in event of an emergency by empowering older users, their families, caregivers, and medicare professionals through remote health surveillance capabilities. Wearable technologies for elderly health surveillance are already making an effect, with total device shipments connected to wearable technologies for elderly health surveillance expected to achieve \$4 million in 2019. Wearables are also becoming the latest means for medical practitioners and health care workers, with ergonomic displays and voice control features for hand-free, computationally assisted quick diagnoses and other health decision making. Wearables may also be employed to create augmented reality for reasons such as better viewing vital organs and tissue during surgery [38]. Wearable sensing device automation is even being employed for environmental surveillance. The capacity to simply monitor plant health, air quality or toxins across a vast region via crowdsourcing is interesting and certain new wearable devices make it even easier. Wearable sensing devices have been researched around the world. Figure 3 shows the number of publications indexed in the Scopus database published in the last four decades (1982-2022) concerning the countries. It can be seen that the U.S.A is leading this research topic with the highest number of publications which is $\sim 2 \mathrm{X}$ higher than the rest of the world (R.O.T.W). 


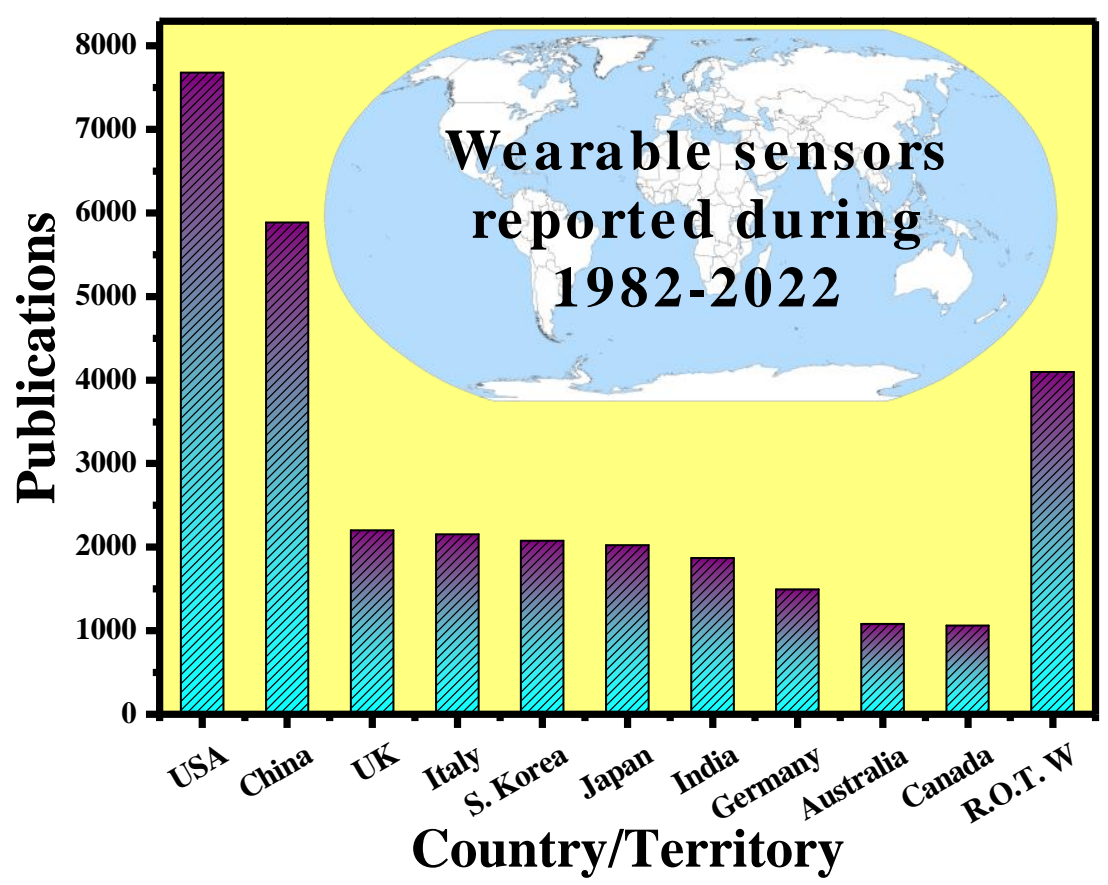

Figure 3. Scientific publication on "wearable sensors (electronics and photonics)" around the world. Results were collected on $19 / 11 / 2021$.

Recent advancements in manufacturing and packaging processes have supported the low-cost embedding of many OE devices and sensing devices on a chip. Furthermore, these chips are extensively employed in many kinds of physiological, biomechanical, and biological sensing, examining, and gathering biomedical data remotely, thanks to a combination of wireless $3 \mathrm{G}$ and $4 \mathrm{G}$ technologies [39]. This has resulted in lower medicare expenditures and enhanced continuous tracking of critical data, particularly for athletes, resulting in increased performance [2]. The development of 5G networks, paired with technologies such as the internet of things (IoT), machine learning, and artificial intelligence, will further revolutionize the future of remote biomedical sensing. Today, medical practitioners use portable diagnostic instruments like glucometers, which offer instantaneous data and are typically unintrusive or less intrusive [40].

However, automation for totally unintrusive blood sugar tracking is currently being developed. As a result, the main goal here is to build unintrusive wearable sensing devices for these sorts of diagnostics that may be employed in uninterrupted tracking procedures. Furthermore, the population is rapidly growing, and physicians are in limited number all around the world. This has compelled the ordinary person to look for alternate choices that would help physicians use their time more efficiently. Individuals can use wearables to trace their important physiological data, with the option to visit a medical practitioner only when necessary [41]. The growing need for personal diagnosis and tracking is a robust sign of the benefits of wearables. Its applications are not limited to tracking blood sugar levels; it has recently been anticipated as a substitute scheme for performing rapid HIV diagnoses [42, 43], timely recognition of Alzheimer's syndrome [44, 45], and perspiration tracking via a wearable paper-based sweat sensing device [46], taking custom-made medicine to a new level [47]. Wearable interfaces such as wearable electronics, electronic skin sensing devices, flexile displays, intelligent robotics, and implanted medical devices, have advanced rapidly in recent years [48]. Due to their unique structure, most of these devices are flexile, portable, adaptable, and easy to use, and they can even be directly bonded to human skin $[49,37,50]$.

There are several non-implantable wearable sensors available for consumer health and medical research, and some devices are currently being used in regular clinical practice. Table 1 lists some of the most prevalent devices [51]. Mechanical, physiological, and biochemical sensors are the three basic types of sensors available. Sensors are available in a variety of grades, from consumer to clinical to research-grade. These sensor's data has 
been utilized for a variety of purposes, including tracking gait, diagnosing atrial fibrillation, and measuring blood glucose, to name a few.

Table 1. Examples of common consumer, clinical and research-grade wearable sensors [51].

\begin{tabular}{|c|c|c|c|c|c|c|c|}
\hline $\begin{array}{l}\text { Manu- } \\
\text { facturer }\end{array}$ & Model & Market & Cost (USD) & Form factor & Sensors & $\begin{array}{l}\text { US FDA } \\
\text { status }\end{array}$ & Ref. \\
\hline Abbott & Libre & $\begin{array}{l}\text { Ambulatory } \\
\text { diabetes } \\
\text { monitoring }\end{array}$ & $\begin{array}{l}149.98 \text { (cost for } \\
\text { reader and } 10 \text { day } \\
\text { sensor) }\end{array}$ & $\begin{array}{l}\text { Semi-inva- } \\
\text { sive }\end{array}$ & CGM & $\begin{array}{l}\text { Ap- } \\
\text { proved }\end{array}$ & $\begin{array}{l}{[52,} \\
53]\end{array}$ \\
\hline $\begin{array}{l}\text { Alive- } \\
\text { Cor }\end{array}$ & $\begin{array}{l}\text { Kardia } \\
\text { Band }\end{array}$ & Consumer & 199 & Wristband & ECG & Cleared & [54] \\
\hline Apple & $\begin{array}{l}\text { Watch Se- } \\
\text { ries } 3\end{array}$ & Consumer & 329 & Watch & $\begin{array}{l}\text { Accel, ambient } \\
\text { light } \\
\text { BALT, } \text { sensor, } \\
\text { PPG Hro, GPS }\end{array}$ & $\begin{array}{l}\text { Precerti- } \\
\text { fied }\end{array}$ & [55] \\
\hline $\begin{array}{l}\text { Ava Sci- } \\
\text { ence, Inc }\end{array}$ & $\begin{array}{l}\text { Ava } \\
\text { Wrist- } \\
\text { band }\end{array}$ & Consumer & 249 & Wristband & $\begin{array}{lr}\text { Accel, } & \text { EDA, } \\
\text { PPG HR, Tem- } \\
\text { perature } \\
\text { sors }\end{array}$ & $\begin{array}{l}\text { Ap- } \\
\text { proved }\end{array}$ & [56] \\
\hline $\begin{array}{l}\text { Bloom- } \\
\text { life }\end{array}$ & $\begin{array}{l}\text { Smart } \\
\text { Preg- } \\
\text { nancy } \\
\text { tracker }\end{array}$ & $\begin{array}{l}\text { Consumer } \\
\text { (rental) }\end{array}$ & 20/week & $\begin{array}{l}\text { Abdominal } \\
\text { patch }\end{array}$ & $\begin{array}{l}\text { Accel, 3-channel } \\
\text { AFE }\end{array}$ & - & [56] \\
\hline $\begin{array}{l}\text { Preven- } \\
\text { tice }\end{array}$ & $\begin{array}{l}\text { Body- } \\
\text { guardian } \\
\text { Heart }\end{array}$ & $\begin{array}{l}\text { Ambulatory } \\
\text { cardiac } \\
\text { monitoring }\end{array}$ & $\begin{array}{l}\text { Ordered through } \\
\text { physician billed } \\
\text { directly to insur- } \\
\text { ance }\end{array}$ & Chest patch & Accel, EFG & Cleared & [57] \\
\hline Oura & Oura ring & Consumer & $299-999$ & Ring & $\begin{array}{l}\text { Accel, Gyro, } \\
\text { PPG HR, Skin } \\
\text { temperature }\end{array}$ & - & [58] \\
\hline Orpyx & $\begin{array}{l}\text { Surro Gait } \\
\mathrm{Rx}\end{array}$ & $\begin{array}{l}\text { Ambulatory } \\
\text { gait moni- } \\
\text { toring }\end{array}$ & $\begin{array}{l}\text { Ordered through } \\
\text { physician }\end{array}$ & $\begin{array}{l}\text { Watch, shoe } \\
\text { insert, shoe } \\
\text { pod }\end{array}$ & Pressure & - & [59] \\
\hline Orpyx & $\begin{array}{l}\text { Surro } \\
\text { Sense Rx }\end{array}$ & $\begin{array}{l}\text { Ambulatory } \\
\text { gait moni- } \\
\text { toring }\end{array}$ & $\begin{array}{l}\text { Ordered through } \\
\text { physician }\end{array}$ & $\begin{array}{l}\text { Watch, shoe } \\
\text { insert, shoe } \\
\text { pod }\end{array}$ & Pressure & Cleared & [60] \\
\hline iRhythm & Ziopatch & $\begin{array}{l}\text { Ambulatory } \\
\text { cardiac } \\
\text { monitoring }\end{array}$ & $\begin{array}{l}\text { Ordered through } \\
\text { physician, billed } \\
\text { directly to insur- } \\
\text { ance }\end{array}$ & Chest patch & ECG & Cleared & [61] \\
\hline $\begin{array}{l}\text { Med- } \\
\text { tronic }\end{array}$ & Enlite & $\begin{array}{l}\text { Ambulatory } \\
\text { diabetes } \\
\text { monitoring }\end{array}$ & - & $\begin{array}{l}\text { Semi-inva- } \\
\text { sive }\end{array}$ & CGM & $\begin{array}{l}\text { Ap- } \\
\text { proved }\end{array}$ & [62] \\
\hline
\end{tabular}

\section{Working mechanism of unintrusive optical sensing devices}

Unintrusive biomedical measures are often conducted optically, with a light source of a certain wavelength $(\lambda)$ being revealed to the area of the skin where the evaluation is desired [63]. The sensing device detects reflected and absorbed light, as well as refracted light, and then characterizes and quantifies the biological data (the identical sensations as employed by spectrophotometer). When transmitting an optical signal through the skin, the $\lambda$ is the most important component since it controls how far the light can penetrate. Depending on the required penetration depth and substantial absorption peak for the relevant sensing application, the $\lambda$ of these light sources can range from UV to deep-IR. The detectors range from broadband photodiodes (PDs) to avalanche photodetectors and photomultiplier tubes. Several illustrations of related passive devices for light capture, $\lambda$ 
selection, and light steering are integrated optics, diffraction gratings, narrowband optical filters, and bulk lenses.

The skin may also be employed as a window to see how the hidden organs are doing physiologically. The use of functional near-IR spectroscopy (fNIRS) to study oxygenation variations in the human brain is one such approach [64]. The current high temporal resolution multi-channel systems perform simultaneous numerous measurements and display the findings in the form of a map or picture across a specified cortical area, employing three separate NIRS methods and complicated data analysis tools. The implementation of multi-channel wearable/wireless devices that allow fNIRS measurements even during regular everyday activities represents the promise that exists for fNIRS more than for any other neuroimaging modality as shown in Figure 4.

\section{High-density system single-trial simulation}

(suitable for multimodal imaging)

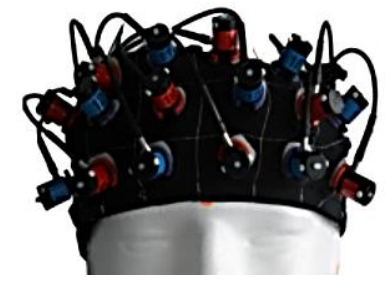

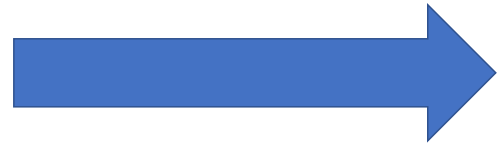

Complex data analysis systems for generating cortical activation maps

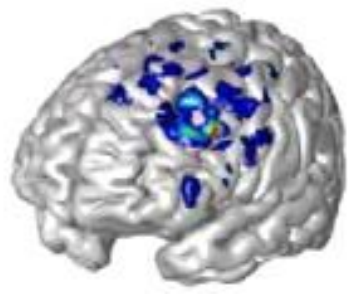

Figure 4. Multichannel fNIRS instrument.

Through its optical interfaces, the skin can sometimes provide a passive conduit for physiological data collecting from hidden vascular systems and organs. As a result, while constructing wearable optical sensing devices, it's critical to consider the skin's optical characteristics [65]. The human skins absorption, transmission, and scattering may be studied by separating the skin into three layers with different optical characteristics:

1) The stratum corneum, which is extremely keratinized owing to the presence of dead squamous cells.

2) The hidden epidermis, which comprises skin pigmentation (mostly melanin) that absorbs shorter $\lambda$, such as UV, and visible (VIS) light to some extent [66].

3) The dermis, which is extremely vascularized and can be described through VIS light and contains carotene, blood hemoglobin, and bilirubin [67]. Because of its thickness relative to the layers above, the dermis attenuates most of the VIS light as seen in figure 5.

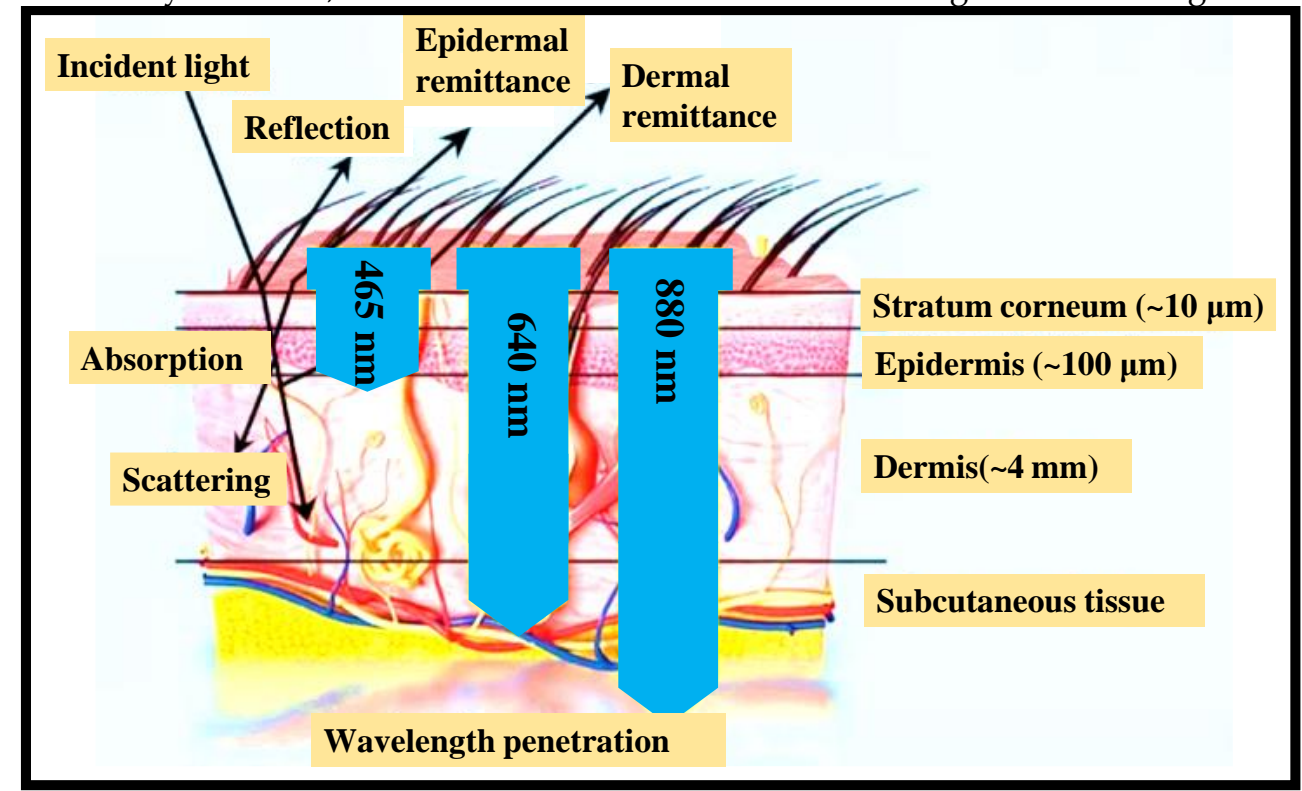

Figure 5. At different sites in the skin layers, incident light displays reflection, absorption, and scattering effects. In terms of $\lambda$, light penetration into the skin [68]. 
Device geometries are determined by the application and evaluation sites on the skin. The light source is mounted opposite the detector in most hard-wired arrangements, as well as traditional wireless devices. This setup guarantees that the detected light interacts with the target tissue across a long optical channel length, resulting in high signal attenuation for pulsatile change extraction [69]. This geometry has the problem of being limited to relevant parts of the anatomy, such as the finger or ear lobe, and it does not provide a simple method for system downsizing [70]. Backscattered reflection approaches allow the light source and detector to be placed near one another in the same plane. As a result, evaluations may be taken via interfaces to practically any part of the body, with minimal downsizing and wireless operation.

Measurements in the reflectance mode, on the other hand, are prone to motion artefacts [71]. In this case, parasitic noise is created by tiny variations in the relative placement of the optical modules to the probing volume. In this context, digital and analogue filtering techniques can be useful [72], and systematic compensatory methods that use accelerometers as motion sensors can offer considerable benefits, albeit at the cost of increased device complexity. As a result, traditional gear for PPG reflection mode measurements is often big and heavy, particularly when wireless operation and power supply are involved. There are additional difficulties in harmonizing the total power utilization and total size of the system with the measurement's signal to noise ratio, where the device current for the light source and the distance between the source and the detector are essential characteristics [73].

\section{Continuous body tracking devices powered by battery}

Optical sensing devices are now commercially available. The most popular optical sensing devices used in wearable electronics are for detecting blood oxygenation and tracking pulse [74]. The absorption and scattering characteristics of light concerning the location of the body described each clinical or biological event when diagnosing it. The oximetry technique, which makes use of variations in the optical characteristics of hemoglobin in its deoxygenated and oxygenated states, is the most visible example [75]. The estimation of critical physiological characteristics, such as pulse inconstancy or variability using photoplethysmography (PPG) and oxygen saturation in arterial blood using pulse oximetry, is possible utilizing analysis of the pulsatile component of the bloodstream [76, 77].

Basic physiological signals include a lot of health-related data. Users are increasingly familiar with the concept of having every step, pulse, and breath recorded and analyzed to offer constant feedback on their health and everyday activities. As a result of this tendency, enterprises are under a lot of pressure to develop accurate, cost-effective, and durable continuous body tracking systems. Wearable optical sensing devices have thus far mostly been utilized for heart tracking [74, 78]. PPG signals have been the focus of several wearable appliances. PPG signals are volumetric measures of an organ, most often subcutaneous blood vessels. The expansion and contraction of arterial volume owing to blood pumping can be observed by variations in optical absorption by lighting a perfuse region of the skin and tracking the reflected or transmitted signal. The pulse rate is consequently determined by the frequency of fluctuating optical absorption (i.e., the AC component of the PPG signal), and the amplitude of the AC component relates to BP in both the systolic and diastolic phases of the cardiac cycle. This method may also be used to test blood oxygenation by using two light $\lambda$ s with differing relative absorption by oxygen-loaded hemoglobin [79]. Mode of transmission when opposed to reflection mode, PPG sensing devices often generate a stronger signal with less motion artefact. However, transmission mode PPG sensing devices have a restricted signal site/location since they must be in areas with high blood profusion, where optical signals have a possibility of travelling to the opposite side of the tissue and being recorded by the receiving PDs.

\subsection{Heart rate monitors (HRMs)}


PPG-based wearable optical heart rate monitors (HRMs) have grown extremely popular, with a slew of tech businesses and products developing and marketing them [80, 81]. Sony, Microsoft, Apple, Motorola, FitBit, MioGlobal, and Masimo, among others, have all developed optical PPG sensing devices that may be worn on the wrist, around the chest, or even in-ear designs using headphone-based optical sensing devices that operate in reflection mode. Ring PPG sensing devices are also of interest because of their small size and potential to produce better signals via transmission-based measurements due to their position on the finger. Commercially accessible sensing devices now perform a wide range of optical and electrical tasks, including measuring PPGs for pulse surveillance as well as blood oxygen levels, tracing the number of steps, breathing rate, temperature (temp.), and even estimating sleep quality [82]. Despite their economic success, there is much debate concerning the exact accuracy and dependability of these gadgets, particularly while doing high-intensity actions. There is no large-scale contributor, peer-reviewed studies that validate the correctness, motion artefact tolerance, and user-to-user variability of commercial wearable HRMs that we are aware of. The strength of the measured PPG signal is strongly location-dependent, limiting the precision and repeatability of the wearable PPG device when consumers do not have the sensing devices securely or consistently attached, which is one of the causes for most of the disagreement [83]. Smith et al. proposed a checkboard-style architecture with alternating OLED and pin PD pixels to overcome this challenge [84]. The PPG signal would be recorded across the array of sensing devices upon startup, and the system would ultimately lock onto the sub-region within the checkboard with the sturdiest signal, reducing DC background noise by turning off OLED pixels not directly adjacent to the perfusion location liable for the robust signal [84].

Athletes are always looking for new technologies and cures to help them improve their health and performance [85]. Athletes are increasingly turning to wearable sensing devices to track their training and recuperation. Athletes' internal and external workloads are presently monitored via wearable devices used by sports teams. The capacity to continuously monitor biomarkers from saliva or sweat in an unintrusive and unintrusive manner remains the next automation gap for sports medicine specialists to personalize hydration and healing programs to the individual athlete [50]. The wearable sensing devices have evolved from a device to a systems perspective during the last two decades, with the system combining the device with data. While earlier research has focused on specific technical domains of the wearables field, such as sensing devices [86, 87], materials $[88,89]$, and soft interfaces [90], or on the production and application of such devices to focus on a particular medical condition, such as atrial fibrillation [91], cystic fibrosis [92], or diabetes [93, 94], there is still an unmet medical requirement to evaluate, develop, and confirm this automation particularly for sports medicine [95].

Optical HRMs functions by beaming light into the body and detecting how light is scattered from blood flow. The technique works best in parts of the body where physiological factors that are not connected to blood flow restrict the quantity of light that is scattered or absorbed. When collecting data from parts of the body with a variety of tissues, such as bone, muscle, tendons, and so on, accuracy may be reduced. Parts of the body undergo greater movement when the body is in motion, such as the wrists and ankles, which have a detrimental influence. There are different spots on the body where wearables can be worn to collect the best pulse as shown in figure 6 . 


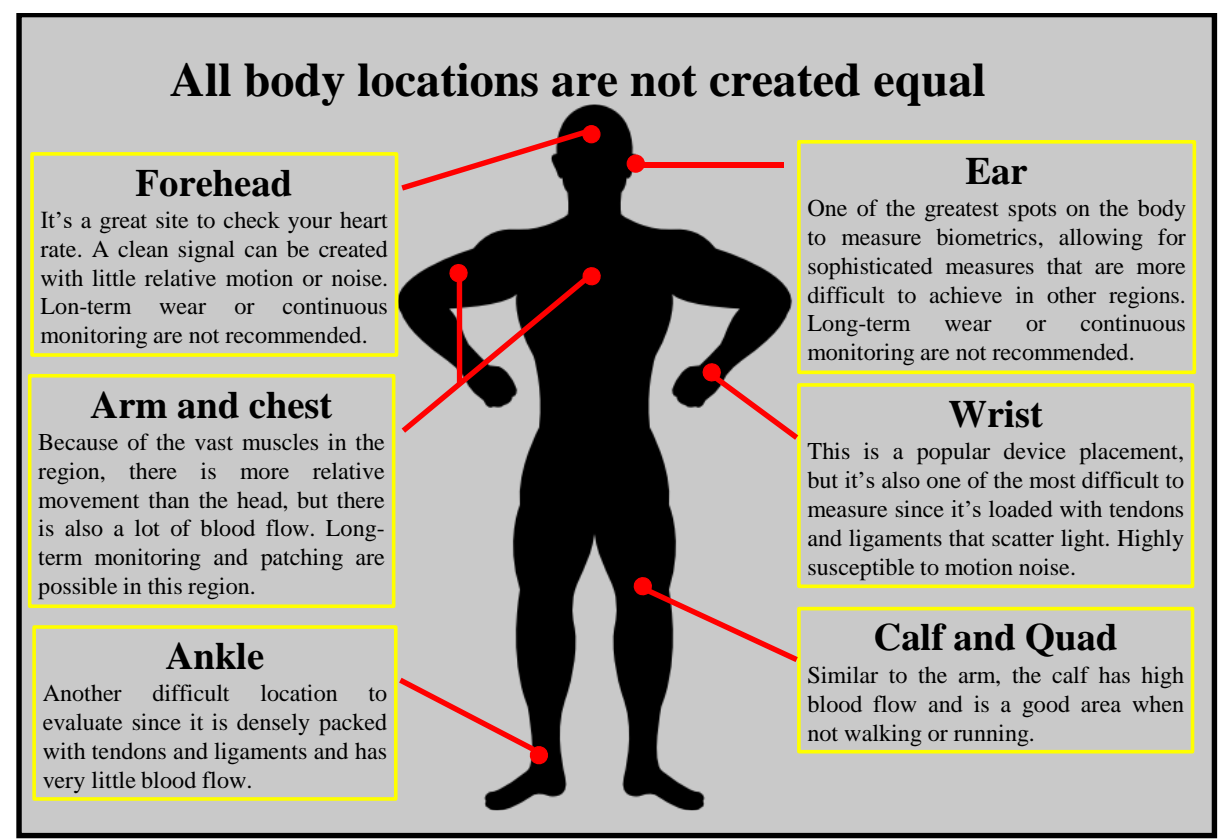

Figure 6. Different possible spots in the body to place HRM to get the best pulse.

While power meters have established the gold standard for gauging effort on the bike, the best HRM may still be a useful addition to your toolkit. It can tell you how your body is performing and how it recovers from exertion on a climb or that additional push is needed to keep a wheel in place. While the traditional chest band is still popular, optical HRMs have begun to gain traction, both as wrist-based sensing devices on the bottom of smartwatches and fitness trackers and as stand-alone devices. For a long time, HRMs necessitated the use of a chest band. Electrodes are put against the skin, and electrocardiography is utilized to record the electrical motion of the heart. HRM chest bands are remarkably accurate, regardless of price, with some devices like the Polar H10 claiming to track the pulse to a granular degree of precision. A chest band had a $99 \%$ correlation with an electrocardiograph, according to a research letter published in [96], while another study published in [97] revealed a Polar H7 to be 99.6\% accurate when tested against an ECG. The pads require a little moisture to pick up the electrical pulses from your heart, so they may take a few minutes to spit out an accurate reading after you start sweating- alternatively, you can lick the electrodes before putting them on for quick reading, but we think that's a little weird. Many individuals have a love-hate relationship with chest-based HRMs since the elastic band is unpleasant and can slip down during a ride. Most employ a detachable pod that holds the hardware to broadcast the ANT+ and Bluetooth signals, as well as a coin cell battery, accelerometers, gyroscopes, and sometimes a little of memory to store running data and even entire activities. Few commercially available HRMs to be worn at different body parts is shown in figure 7 (a-d).

Additionally, all-optical approaches for tracking the heart cycle in an MRI have been proposed. Rothmaier et al., for example, showed the application of photonic textiles for pulse oximetry measurements [98]. The researchers were capable of recovering a PPG in transmission mode when the finger was lit by a peripheral light source of $\lambda=690 \mathrm{~nm}$ and $830 \mathrm{~nm}$ by weaving polymer optical fibers (POFs) into the forefinger of a glove. The modified Lambert-Beer law was utilized to compute arterial oxygen saturation using this dual$\lambda$ light. Utilizing an amalgamation of coupling augmentation methods for instance roughening the fiber surface, adding fiber back reflectors, and strategic fiber cuts in the direction of the incident light, it is possible to optimize the POF incorporation of diverse weaving and embroidered conformations to realize nearly 100 times enhancement in coupling competences over the unaltered woven POF fibers. This unique technique is appealing, but it still must be enhanced in words of coupling effectiveness to get a high SNR in the PPG signal, as well as solve the same key issues related to motion artefacts that traditional PPG optical sensing devices encounter. 


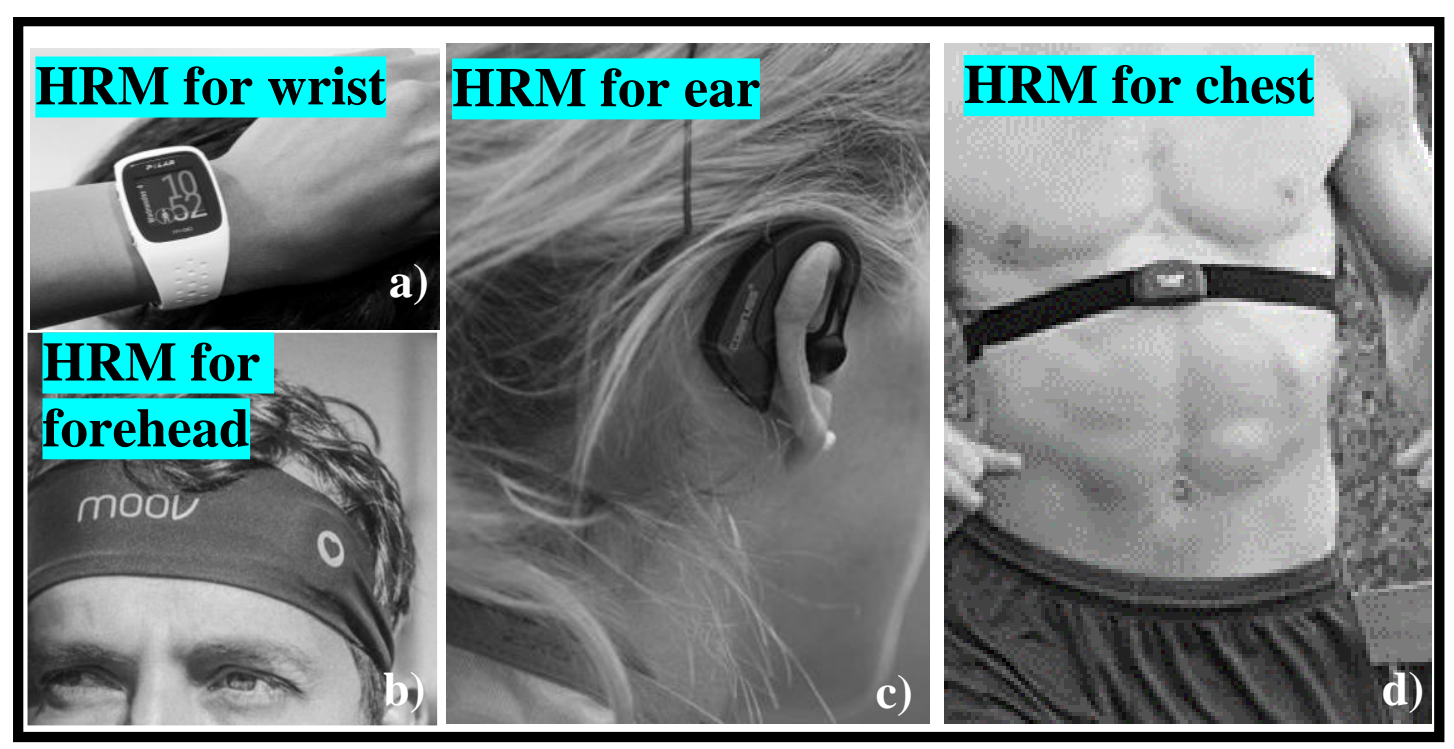

Figure 7. Commercially available HRM, a) wrist [99], b) forehead [100], c) ear [101], d) chest [99].

\subsection{Continuous Glucose Monitoring (CGM)}

Diabetes is a disorder that affects around 415 million people worldwide. The most prevalent kind of diabetes is type 1 diabetes, which occurs when the body attacks the cells in the pancreas, preventing it from producing insulin [102]. Type 2 diabetes, on the other hand, occurs when the body is incapable to generate enough insulin or when the insulin that is produced is ineffective [103]. Although diabetics may live healthy, happy, and productive lives, maintaining blood sugar control is an important component of treating the disease. To do so, it is required to keep a close eye on the blood sugar levels. The advancements in medical automation have been astounding since the first blood sugar test strip was produced in 1965 [104]. CGM was established as a means of regulating blood sugar levels in 1999, and it was one of the most significant advancements in the history of blood sugar tracking. Since 1999, CGM has grown in sophistication, with new products always building on the development of previous ones. Flash continuous glucose monitoring (FCGM) is one of the most notable new kinds of CGM [105, 106].

Self-monitoring of blood sugar remains an extremely prevalent method of glucose monitoring (SMBG) [107]. This entails pricking the victim's finger and drawing a little blood sample to determine the amount of blood sugar (figure 8, left). After then, the victim gives insulin or glucose as needed. This strategy, while simple, has certain disadvantages. Victims may find SMBG inconvenient since testing might be uncomfortable. It is described as antisocial by many people, and it is influenced by daily living activities. This can be problematic, especially for younger individuals. Importantly, for this approach to be effective, regular testing is necessary, which reduces its attractiveness for consumers searching for discreet and simple ways to test their blood sugar levels [108]. An implantable glucose sensor is also used in clinics (figure 8, right) [109]. The embedded sensor is more intrusive and sensitive to biofouling, but it provides CGM. Furthermore, because it detects glucose in the intravenous blood rather than the peripheral blood, an implanted sensor improves the accuracy and clinical usefulness of the data. Even though various bloodbased monitoring methods have been devised, diabetic patients do not always follow the protocol due to the discomfort and difficulty connected with the intrusive detection procedure. In the case of Type I diabetes, a considerable percentage of pediatric patients are particularly averse to the needle-pricking phase required for blood-based glucometers. It is critical that a painless and noninvasive approach for monitoring glucose levels be developed.

CGM, on the other hand, allows victims to monitor their blood sugar levels at all times using a wearable device that gathers data on the body's blood sugar levels. CGM devices are available 24 hours a day and incorporate alerts that sound when a victim's blood sugar levels are abnormally high or low [21]. Finger pricking is no longer required 
or is much reduced. This is beneficial to the victim's health and decreases the possibility of overstating. Furthermore, CGM enables individuals to analyze data to spot trends and patterns, which may then be shared with their medicare providers.

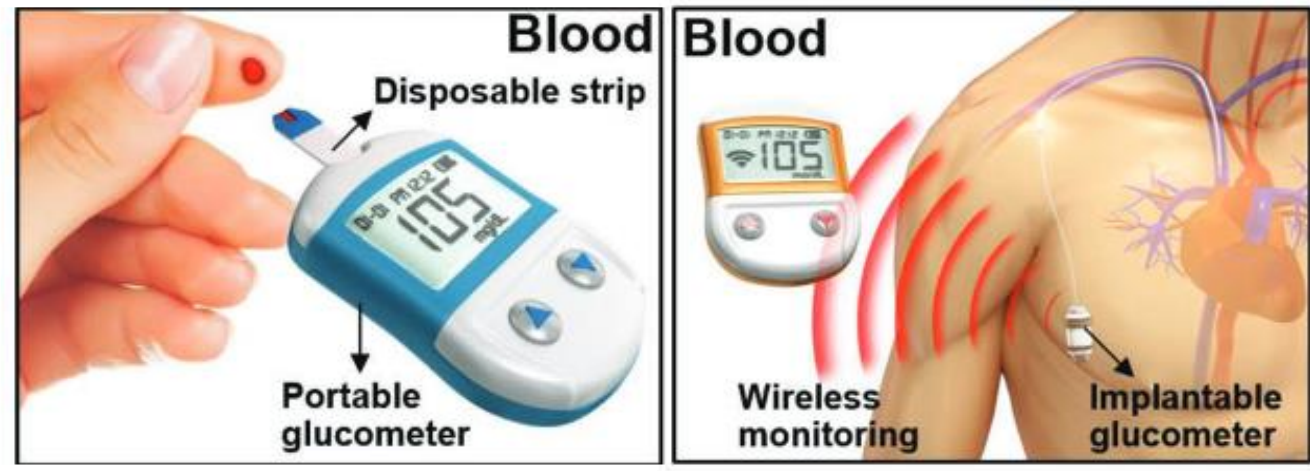

Figure 8. Invasive glucose monitoring systems [109].

While research into novel technologies for miniaturizing electrochemical biosensors is still ongoing, other techniques established on optical sensing have become far more enticing recently [110]. Optical sensing technologies can circumvent many of the limitations associated with electrochemical sensing devices, such as sensing device sensitivity and stability being dependent on the enzyme utilized and interference with active substances (acetaminophen, ascorbate). Numerous optical recognition approaches have been proposed in the literature, including near-IR detection and Raman spectroscopy for unintrusive detection and fluorescence-based sensing devices for implanted systems [111]. Senseonics (Senseonics, Inc., Germantown, MD, USA) has recently successfully applied this last category of optical sensing technologies to develop the Eversense sensor, a fully implanted CGM system [112] that provides real-time blood sugar measurements through an external coupled transmitter for an expected lifetime of 6 months [113]. With a lifespan of 90 days and an accuracy of $11.4 \%$ MARD, the Eversense CGM is currently only certified for usage in European nations (CE mark acquired in 2016) [114]. While biocompatibility and victim acceptance of the new completely embedded automation stay major concerns, the approach's primary virtues are its longevity and ease of use.

The sensing devices (which sits in the skin), the transmitter (which sits above the skin), and the receiver (either smartphone or a handheld device that receives blood sugar data) are the three main components of most CGM sensing devices. The majority of CGM sensing devices (very thin wire-like components) are implanted under the skin (body fat) [115]. Typically, the sensing device is implanted with a tiny needle using a simple at-home application device. The needle retracts almost immediately, leaving the sensing device in situ. The transmitter, which rests on top of the skin and is held in place by an adhesive, is the most visible part of a CGM. CGM sensing devices can be put in a variety of locations on the body, depending on the user's preference: back of the arm, side/front torso, lower back, buttocks area, outer thigh, and so on. Avoid areas where the body bends a lot naturally, or where clothes can irritate or be a nuisance. Inserting the CGM sensing device is a simple and almost painless procedure.

A blood sugar sensing device has been designed that is both cost-effective and extremely wearable, with a short data gathering time frame that allows for an unintrusive, long-term CGM system [116]. During the variation of blood sugar conc. at the wrist tissue, the innovative biosensing device exploits unique data of the pulsatile to continuous components of the arterial blood volume pulsation. The combined VIS-NIR spectroscopy was used to measure the reflected optical signal.

NIR light may penetrate multiple layers of the skin and get to the arteries in the subcutaneous tissue, as illustrated in figure 9a, but VIS light, such as green and red light, can only make it to the capillaries and arterioles in the dermis tissue. Consequently, by collecting reflected light in different optical $\lambda$ s at varying levels of blood sugar conc., several spectra data may be acquired that can be used for multivariate analysis. As shown in figure $9 \mathrm{a}$, NIR-light can penetrate multiple layers of skin to reach the arteries in the 
subcutaneous tissue, but VIS light, such as green and red lights, can only reach the capillaries and arterioles in the dermis tissue [116]. As a result, by collecting reflected light in various optical $\lambda$ s at various levels of blood sugar conc., we will have a variety of spectral data that can be used in multivariate analysis. As illustrated in figure $9 \mathrm{~b}$, the suggested prototype acquires data via analogue signal filtering.

The sensing device system is affixed to the wristband 'rear side and is in direct touch with the subject's skin tissue. It is situated between the interosseous arteries on the subject's outer wrist, with an overall sensing device size of $15 \times 15 \mathrm{~mm}^{2}$, as shown in figure 9c and figure 9d, respectively. An SFH7060 (OSRAM semiconductor Inc., Germany) multichip sensing device package with dimensions of $7.2 \times 2.5 \times 0.9 \mathrm{~mm}$ is used to transmit IR LED light of $950 \mathrm{~nm}$, Red LED light of $660 \mathrm{~nm}$, and Green LED light of $530 \mathrm{~nm}$, and to identify the reflected light utilizing an integrated PD with a spectral range of $400 \mathrm{~nm}$ to $1100 \mathrm{~nm}$. Furthermore, for 850 nm, a VSMY2853 GIR LED (Vishay Intertechnology Inc., USA) with a 100-mA forward current is employed. As a result, two VIS-LEDs and two NIR-LEDs are employed in total.

The median association coefficient between the approximate and reference blood sugar conc. was 0.86 , with a standard prediction error of $6.16 \mathrm{mg} / \mathrm{dl}$, in an in-vivo experiment including 12 participants in a 2-hour controlled carbohydrate-rich diet. Furthermore, the suggested sensing device's dependability was tested for a whole day. The results revealed that the model constructed the day before could accurately predict a fullday blood sugar conc. that was measured the next day.

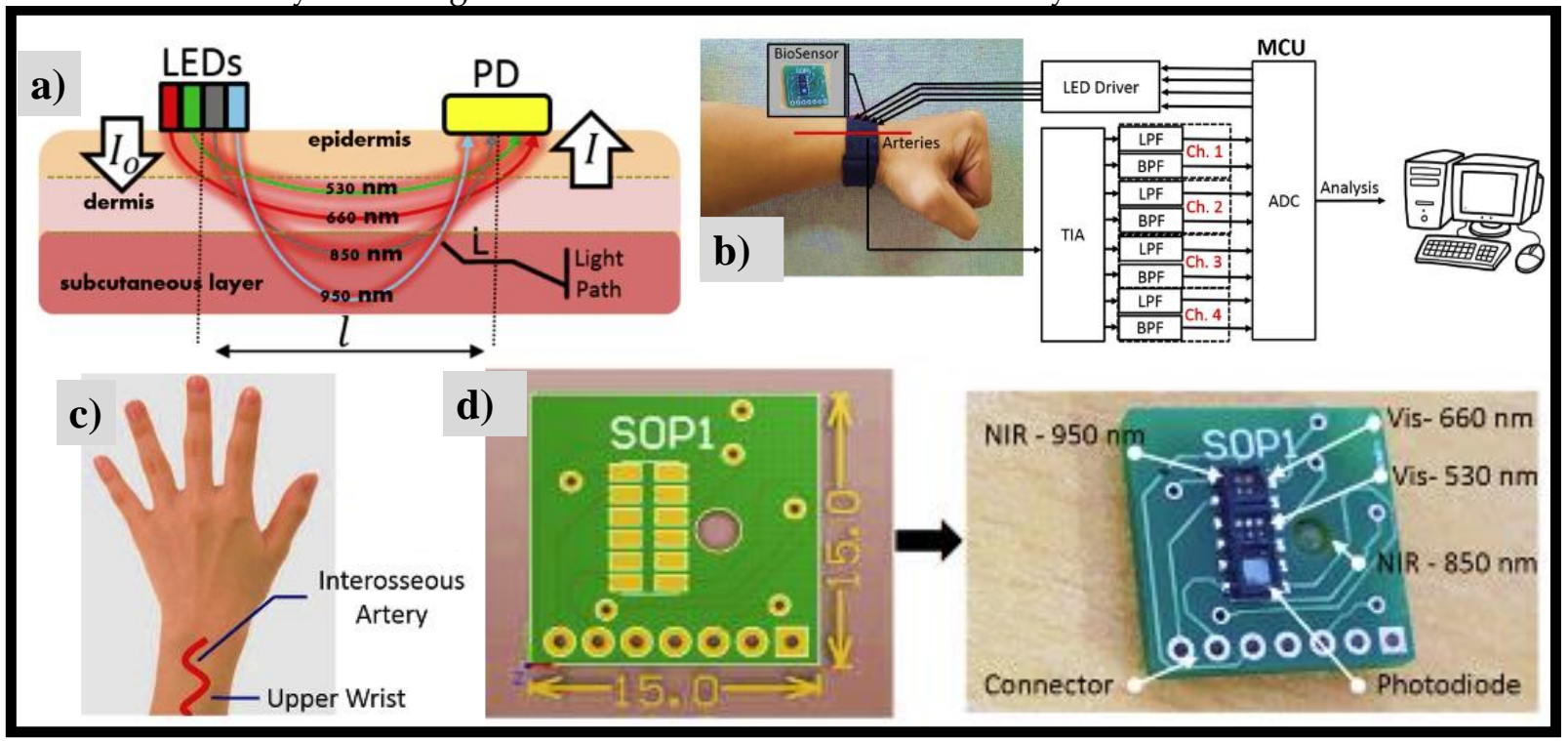

Figure 9. a) Diffused-reflectance PPG signal in multiple $\lambda$ measurements [116]. Prototype system: b) block diagram of analogue signal processing [116], c) sensing device location wear on the wrist [116], d) the overall sensing device design on PCB [116].

\section{Skin-like wearable sensing devices}

In the biomedical investigation, clinical treatment, and remote diagnostics of health state, accurate tracking of temp., BP, oxygenation, blood flow, and electrophysiology are critical [117, 118]. Most commercially available wearable gadgets are mechanically hard components attached on bands and worn on the wrist, with form factors eventually constrained by the size and weight of the batteries required for the power supply. Wearable gadgets with "skin-like" qualities are a new kind of automation that is only starting to make its way out of research labs and into pre-commercial prototypes [119, 120]. The use of near-field communication (NFC) automation for multicolour light emission and recognition in a way that permits accurate evaluations of the optical features of the skin, to detect peripheral vascular disease (PVD) and assess colouration, and/or of colour-responsive materials for environmental discovery is demonstrated in several battery-free, wireless OE devices.

In biomedical research, clinical care, and remote diagnostics of health condition, accurate tracking of temp., BP, oxygenation, blood flow, and electrophysiology is critical. 
Commercially available wearable gadgets are mostly mechanically hard components placed on bands and worn on the wrist, with form factors restricted by the size and weight of the batteries required for the power supply. Wearable gadgets with "skin-like" characteristics represent a new kind of automation that is just now making its way out of research labs and into pre-commercial models. These gadgets take advantage of materials and layouts that result in system-level physical characteristics that are like those of skin.

\subsection{Skin-electronics interface}

Wearable sensor integration must consider not just flexible and biocompatible materials, but also intimate contact forms on the human epidermis. Here, three types of skin interfaces are examined: tattoo-like, medical bands, and textiles.

\section{i. Tattoo}

Tattoo-like wearable sensors represent an emerging class of ideal device shapes, with their conformal and practically invisible look. Many critical signals, such as the electrocardiogram (ECG), electromyogram (EMG), and electroencephalogram (EEG), can be accurately monitored using tattoo-like sensors since intimate physical contact may be obtained [121]. In addition, chemical sensing is available in this format. Tattoo-based noninvasive lactate monitoring was proven in a typical application by analyzing sweat during cycling activity with an enzymatic biosensor. A lightweight and breathable kind of onskin electronics that bonded tattoo-like conductive nanomesh directly onto human skin was presented to improve gas permeability and wearing comfort [122]. Tactile sensing was studied a long time without generating inflammation.

\section{ii. Band}

Alternative technologies for on-skin applications are required for sophisticated sensor systems that include stiff components like as integrated circuit chips. Medical bands are frequently utilized to make direct touch with the skin without causing undue pain. To continually monitor he pulse wave of the radial artery, a polymer transistor-based pulse sensor may be readily fixed on skin using a standard bandage [123]. A completely integrated wristband with a chemical sensor array and FPCB was recently created for in situ detection of sweat metabolites and electrolytes [124]. Similarly, a graphene-based band platform applied to the forearm was used to monitor glucose levels in the interstitial fluid in real time [125].

\section{iii. Textile}

Wearable textile sensors can be sewn into clothes or knitted into them for long-term use. Conductive textiles may be used to make functional electrical components including antennas, resonators, and pressure sensors [126]. Sensor textiles for skin interfaces can be developed as clothing or gloves. Textile wearable devices are commonly used as an appropriate platform for energy-harvesting applications owing to frictions with the human body. Clothing with a triboelectric energy generator (TENG) and supercapacitors are recently developed [127]. Energy collecting and storage were shown using a natural armswinging action while walking. With skin interfaces, the main goal is to create close skin contact and capture high-quality sensory data. Rigid electronics create gaps between the device and the skin, resulting in artifacts and noise while moving. Meanwhile, many tattoo-based sensors still require additional power and analysis devices, and more research combining low power consumption and totally flexible skin electronics is underway [128].

\subsection{Materials and structural designs}

The mechanical qualities of wearable sensing devices must be soft, flexile, and compliant to curved skin for them to be integrated with the human body. Silicon is the most common semiconductor material, although its modulus is $1 \times 10^{5}$ times greater than that of human skin. Given the significant mechanical mismatch, the most logical solution is to 
produce naturally soft and conductive materials to provide mechanical compatibility and increased wearing comfort. Soft electronic materials for skin applications have a lot of potential because of recent advances in materials science. However, there are still issues with these newly produced materials. While liquid conductors [129] such as gallium metal alloys are low in toxicity and activity and can give unlimited stretchability, most liquid conductors require microfluidic channels to deliver elastomer for skin sensing devices [130]. Although hydrogels [131, 132] and polymers [133] are very biocompatible, their effectiveness is restricted by their poor conductivity, and many hydrogels dry up and harden with time. To improve performance and stability, current biocompatible materials are mainly made up of composite materials that include hydrogels, polymers, and nanoparticles [134]. To avoid any health risks, caution should be used while employing nanomaterials such as carbon nanotubes encapsulated inside elastomers [135, 136]. Biocompatible materials need to be developed further for improved stability and breathability in practical applications [137].

Polydimethylsiloxane (PDMS), polyethylene terephthalate (PET), polyurethane (PU), polyimide (PI), polyethylene naphthalate (PEN), polyethylene oxide (PEO), polystyrene sulfonate (PEDOT: PSS), Ecoflex, and other materials are commonly used as flexile metal foils or non-metallic substrates in flexile skin-like sensing devices [138]. Various polymers were found to be appropriate for use as flexile substrates in the creation of flexile devices. The use of PDMS and transparent electrodes is proposed for a flexible pressure sensor as shown in figure 10 (a-c). The whole device has an $82 \%$ transmittance and a minimum bending radius of $18 \mathrm{~mm}$. Apart from that, the impact of annealing temperature on the mechanical characteristics of PDMS is discussed in [139]. The results demonstrate that the PDMS film has acceptable compression properties but poor dynamic responsiveness at a lower annealing temperature of $80^{\circ} \mathrm{C}$. The compression property of PDMS films was dramatically diminished at higher temperatures. The annealing temperature of around 110 ${ }^{\circ} \mathrm{C}$ is determined to be the optimal compromise between compression property and dynamic responsiveness for PDMS film. The pressure sensor has an excellent sensitivity of $0.025 \mathrm{kPa}^{-1}$ and a strong response property when cured at $110{ }^{\circ} \mathrm{C}$. The invention paves the way for intelligent transparent sensing applications in the future.

Furthermore, MXene-based hybrid materials with superior characteristics have a lot of potential in next-generation pressure sensing devices for a wide range of applications [140]. The fast growth of graphene and other 2D material syntheses, as well as new manufacturing methods for functional devices, has alleviated some of the problems associated with traditional materials, allowing for the application of emerging wearable flexile electronics and complex functional products [141]. The chemical structures and characteristics of illustrative biopolymers outlined the design and production strategies for biocompatible conductors established on these biopolymers are demonstrated and highlighted production methods for several biocompatible conductors for flexile bioelectronics [142].

Lei et al. created a wearable biosensor based on MXene for sweat analysis as shown in figure 10d [143]. The device was created by combining 2D MXene $\left(\mathrm{Ti}_{3} \mathrm{C}_{2} \mathrm{~T}_{\mathrm{x}}\right)$ nanosheets with a $\mathrm{Ti}_{3} \mathrm{C}_{2} \mathrm{~T}_{x} / \mathrm{PB}$ composite and enzyme to create an oxygen-rich enzyme biosensor for $\mathrm{H}_{2} \mathrm{O}_{2}$ detection. $\mathrm{Ti}_{3} \mathrm{C}_{2} \mathrm{~T}_{\times} / \mathrm{PB}$ composites demonstrated higher electrochemical performance toward $\mathrm{H}_{2} \mathrm{O}_{2}$ detection than carbon nanotubes/ $\mathrm{PB}$ and graphene/ $\mathrm{PB}$ composites due to the high conductivity and strong electrochemical activity of exfoliated MXene. The sensing device has a changeable sensor component that may be inserted and replaced with customized sensors to track various analytes, such as glucose, lactate, or $\mathrm{pH}$ value. The device's substrate was made of superhydrophobic carbon fiber, which was utilized to establish a tri-phase contact and protect the connection against sweat corrosion. Artificial perspiration was used to test the as-prepared device's sensing capabilities. In addition, the sensor was put to the test on human participants and was utilized to analyze perspiration. The results revealed great sensitivity and reproducibility in simultaneous glucose and lactate readings.

Electronic systems may be utilized in a conformal manner on curved and soft surfaces thanks to mechanically deformable devices and sensors. Because liquids are 
intrinsically more flexible than solids, sensors that use liquids enclosed in soft templates as the sensing component provide an appropriate platform for such applications. However, because of the difficulties in fabricating liquid-based junctions due to intermixing, liquid-based devices have been confined to metal lines based on a single liquid component. A stable foundation to produce liquid-liquid heterojunction devices paving the way for liquid-state electrical systems to become a reality. The device design and manufacturing strategy are general for various detecting liquids, allowing for the demonstration of sensors that respond to various stimuli. A temperature, humidity, and oxygen sensors are developed as a proof of concept employing various ionic liquids, displaying great sensitivity, and outstanding mechanical deformability due to the intrinsic characteristic of the liquid phase as shown in figure 10e [144].

The researchers created a biotransferrable graphene wireless nanosensor for bacteria detection in saliva [145]. The sensor's design is shown in figure 10f. A graphene-based sensing element with a wireless readout coil was first created on silk fibroin. Second, the graphene-based transducer was biotransferred onto a tooth surface before the supporting silk film was dissolved as depicted in figure $10 \mathrm{~g}$. After that, self-assembling antimicrobial peptides were applied to the graphene monolayer to increase detection specificity. When the target bacterium is recognized and bound, the graphene film's electroconductivity is modified and wirelessly monitored using an inductively connected radio frequency reader device (figure $10 \mathrm{~h}, \mathrm{i}$ ). The created hybrid nanosensor was shown to have a low detection limit (100 CFU/mL), operate without batteries, and be capable of distant wireless sensing.

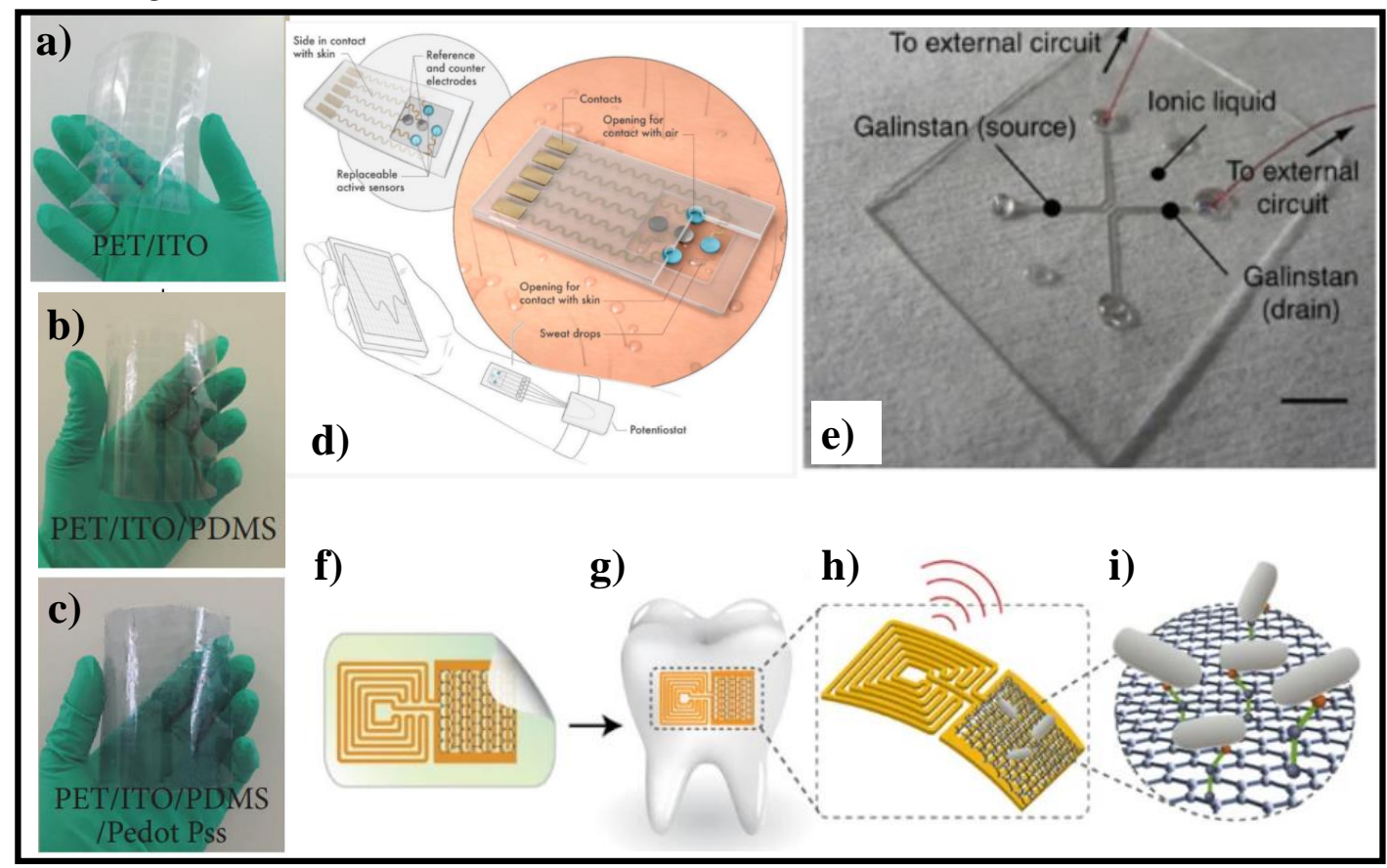

Figure 10. Flexible substrates for biosensors, a-c) PDMS and PEDOT:PSS based pressure sensor [139], d) wearable sweat sensor based on MXene [143], e) Fully fabricated liquid heterojunction device consisting of an ionic liquid active channel and GaInSn source/drain electrodes [144], f) graphene based wireless nanosensor [145], g) biotransfer of the nanosensing architecture onto the surface of a tooth [145], h) Magnified schematic of the sensing element, illustrating wireless readout [145], i) Binding of pathogenic bacteria by peptides self-assembled on the graphene nanotransducer [145].

\subsection{Communication and data analysis}

Near-field communication (NFC) is new automation that permits two electronic devices to communicate by merely placing them close together [146]. For chloride detection, for instance, NFC supported thermography was paired with colourimetric microfluidic sensing devices [147]. An NFC chip was coupled with an onboard temp. sensing device to provide real-time sweat loss and electrolyte conc. diagnostics. NFC transmission, for example, permits not just data but also power transfer. A hybrid skin-interfaced device with electrochemical, colourimetric, and volumetric sweat analysis capabilities was 
recently introduced [148]. A tiny NFC electronic module and a microfluidic system make up the battery-free arrangement. The system is started by bringing an NFC-supported portable device close enough to capture wireless, real-time data as well as digital photos for colourimetric analysis [148]. Despite its lightweight and low power utilization, NFC automation has an extreme delivery distance of about 4 inches and requires the antenna to be kept in a specific orientation, as well as a proximal power source placed close to the subject, limiting the subject's range of activity, and making it unsuitable for long-term use.

Sweat can be used to provide unintrusive, in situ biochemical tracking of physiological state, which might lead to new kinds of medicare diagnostics and individualized hydration methods. Recent developments in sweat collecting and sensing automation are attractive, however, they are ineffective in extreme circumstances like aquatic or arid environments. Eliminating interference/contamination from surrounding water, maintaining robust adhesion during viscous drag forces and/or vigorous motion, and limiting the evaporation of collected perspiration all provide distinct problems. The materials and design for waterproof, epidermal, microfluidic, and electrical devices that stick to the skin, store, and analyze sweat even while fully submerged are discussed [147].

The device platform is made up of a waterproof combination of skin-like, or epidermal, microfluidic, and electronic arrangements that are laminated onto the skin for sweat collection, storage, and chemical analysis, as well as digital skin temp. evaluation in both aquatic and dryland environments, including the transition between the two. Microchannels, a chamber containing a colourimetric chemical reagent, electronics for wireless communication and precise temp. tracking, a set of reference colour markers, and a skin-safe glue are all included in the structures as shown in figure 11A [147]. The molded layer of poly (styreneisoprene-styrene) (SIS) attaches to a thin, flat sheet of SIS to define the sealed microfluidic arrangement and enclose the colour markers as shown in figure 11B [147]. The colourimetric reagents are either a food dye for visual assessment of the degree of sweat filling of the $\mu$-channel or a silver chloranilate solution that interacts with sweat to give a colourimetric response proportional to the chloride conc. This reagent is kept in a compartment next to the intake, where it reacts when perspiration enters the channel as shown in figure 11C [147]. As the device fills with perspiration, the food dye comprises red and blue water-soluble particles with differing dissolving rates, resulting in a volumedependent colour gradient as shown in figure 11D [147]. A wireless interface to NFCsupported devices for transmitting digital identification codes and reading skin temp. is formed by a flexile magnetic loop antenna, a set of NFC components, and a LED as a mode for user notification. The NFC chips are encased in SIS, which permits for long-term operation even when the gadget is immersed in water as shown in figure 11E [147]. The LED emits light through the microfluidic layers during wireless operation in a wet environment as shown in figure 11F [147]. 


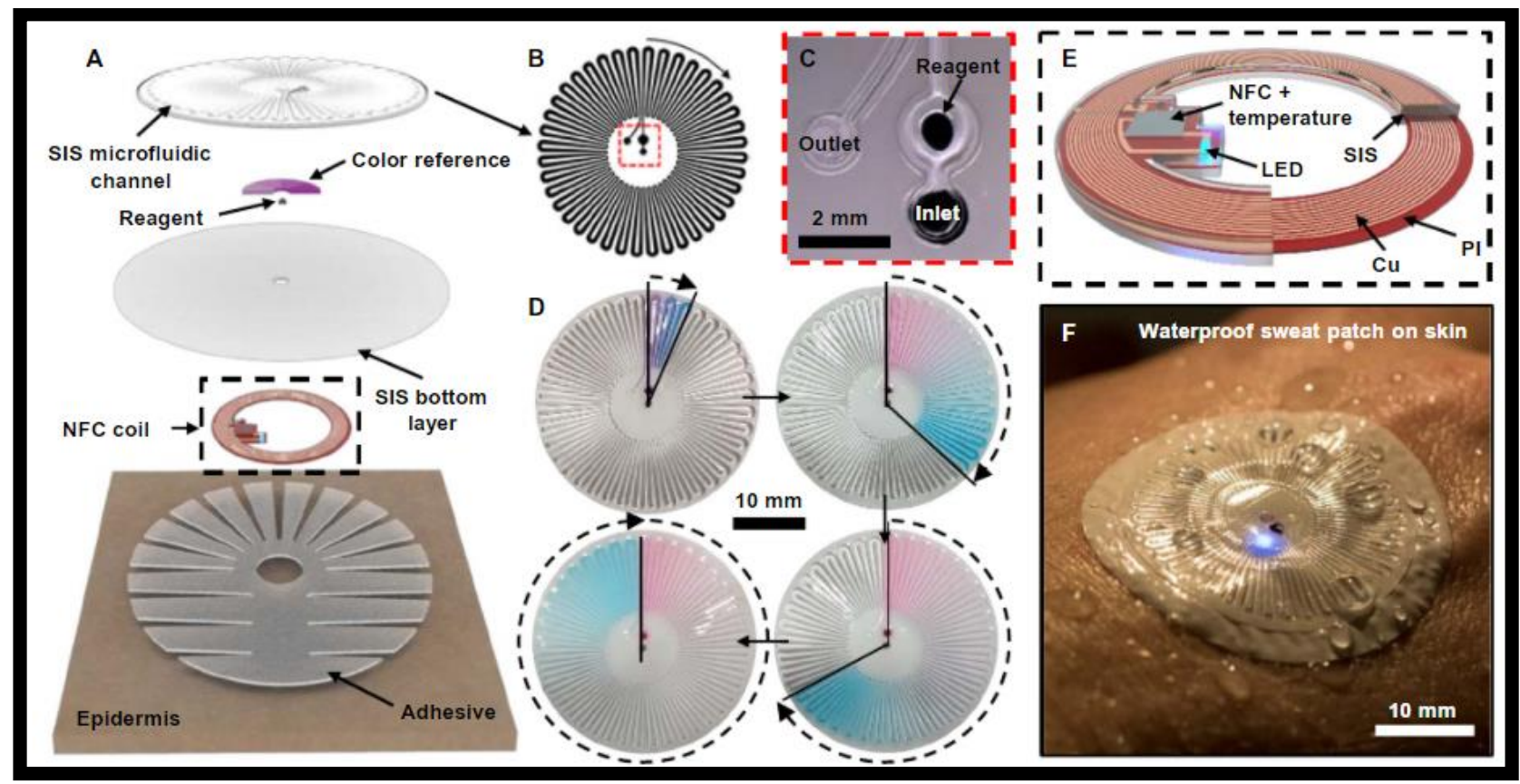

Figure 11. Skin-like microfluidic/electronic device that is waterproof, A) Schematic depiction of the major layers of a typical device [147], B) geometry of microfluidic channels [147], C) optical micrograph of the colourimetric reagent and microfluidic intake and outflow ports [147], D) A flow-driven colour shift is produced by a dye made up of blue and red water-soluble particles that dissolve at different speeds. The total volume of collected perspiration is calculated by counting the number of turns of filled tubes [147], E) NFC coil for wireless evaluations of skin temp. [147], F) Sweat gathering in aquatic environments without contamination is supported by small outlet geometries and constituent polymer materials that are hydrophobic and largely impermeable to water and water vapour. Underwater functioning of the electronics, containing the NFC coil, integrated circuit chip, and indicator LED, is supported by dip-coating an encapsulation of the same material [147].

For wearable medicare, Bluetooth low energy (BLE) provides an alternate communication platform [149]. In comparison to NFC, BLE offers a quicker communication rate and a reading distance of over 30 feet, making it ideal for multipurpose sensing and longterm use. Integrated devices comprising of a rechargeable battery, a microcontroller, signal-processing circuits, and a Bluetooth transceiver were constructed on a flexile printed circuit board (FPCB) board and coupled to a flexile electrochemical sensor in a typical configuration of an electrochemical sensor arrangement [124]. Bluetooth captures data in real-time, sends it to the user's phone, and then uploads it to cloud servers. The mechanical rigidity of a BLE module, on the other hand, is undesirable for skin interfaces, and most commercially available BLE modules consume milliwatts of power, implying high power needs.

The frequency of RF transmission is set at $13.56 \mathrm{MHz}$, and it achieves coupling between an initiator and a passive target [150]. A chip-free and battery-free on-skin sensor network that uses RF identification automation to communicate wirelessly with flexile readout circuits mounted to textiles is reported in [151]. To establish a conformal skin contact, the elastic sensors were made of inherently soft materials without any hard silicon circuits or batteries, whereas the initiator circuit incorporated a Bluetooth transceiver for data transfer to a smartphone. The device was shown to continually track pulse, respiration, and body movement. Wireless communication and data analysis advancements have permitted increased shrinking and incorporation of wearable sensor arrangements, however, significant restrictions remain. NFC and RF communication can be made completely flexile and conformal to the skin, but they have a small range of operation and need antenna-readout electronics to be positioned near the sensors. BLE permits for quicker and more efficient data transfer, but it requires specific integrated circuit chips that cannot be produced flexibly at this time. Because wireless communication modules consume a significant amount of power, they must be paired with power management to provide a fully integrated sensor platform.

\subsection{Applications of skin-like sensors}


Several battery-free, wireless OE devices are demonstrated that utilize near-field communication (NFC) automation [152] for multicolour light emission and recognition in a way that permits specific evaluations of the optical attributes of the skin, to detect PVD and estimate colouration, and/or of colour-responsive materials for environmental recognition [153]. Explicit cases comprise devices that can monitor pulse, tissue oxygenation, pressure pulse dynamics, UV exposure, and skin colour through an integrated collection of time-multiplexed miniaturized LEDs and photodetectors whose signals are amplified and digitized before wireless broadcast [154, 155]. Form factors proficient of soft and conformal lamination onto the skin, as well as the capacity to operate effectively under substantial strain (up to $30 \%$ uniaxial) deformation, are the result of carefully optimized materials and mechanical designs. These findings point to a basis for automation that may be used in both medicare and non-medicare applications. These systems are significant advancements over devices that rely on wired data broadcast and traditional power sources to evaluate arterial blood oxygenation using commercial or organic LEDs and photodetectors mounted on flexile, rather than elastic supports [98, 156].

Flexible skin-like sensing devices have accomplished numerous functionalities previously inaccessible for standard sensing devices due to their deformability, lightness, portability, and flexibility. Physical, chemical, physiological, and multifunctional flexile skin-like sensing devices are the four primary kinds of flexile skin-like sensing devices depending on the quantities they detect. Physical sensing devices are created by utilizing the physical characteristics of the substances that are susceptible to the measured quantities [157]. Chemical sensing devices are made up of responsive components that transform chemical quantities into electrical quantities, such as the composition and conc. of chemical compounds. Physiological sensing devices are sensing devices that detect and recognize biological and physiological quantities in living organisms by using different biological and physiological features or characteristics of biological substances. Pressure, strain, and temp. are the three most common physical variables that may be sensed by flexile skin-like physical sensing devices, which can transform external physical stimulus signals like pressure, strain, and temp. into electrical impulses, completing the skin's sensing function [158]. Multifunctional sensing devices are made up of several distinct kinds of sensing devices combined into a single unit that can identify and test multiple variables instantaneously [159]. It has been stated, for example, that the multifunctional electronic skin can monitor pressure, tension, and temp. all at the same time [160]. Flexile sensing devices have a wide range of categories and uses, thus worldwide research on them has accelerated in recent years [158].

A novel design of a temp. sensing device is proposed which is made of cross-linked poly (3,4-ethylenedioxythiophene): poly (styrenesulfonate) (PEDOT: PSS) [161]. Significant improvements in humidity stability and temp. sensitivity of PEDOT: PSS based film were achieved by combining the crosslinker (3-glycidloxypropyl) trimethoxysilane (GOPS) with the fluorinated polymer passivation (CYTOP). The fabricated sensor device showed outstanding stability in a range of ambient humidity from $30 \%$ to $80 \%$ relative humidity, as well as high sensitivity of $-0.77 \% /{ }^{\circ} \mathrm{C}$ for temp. detection between $25^{\circ} \mathrm{C}$ and $50{ }^{\circ} \mathrm{C}$. The schematic diagram of a wireless sensing platform mounted on an arm for realtime body temp. tracking is shown in figure 12a [161].

The most often utilized sensing devices for body detection are strain and pressure sensing devices. With countless papers, the discipline is fast evolving and prospering [162, 163, 164]. The mechanism, structural compositions, performance quantities, and applications of pressure and strain sensing devices vary widely. A new kind of sensing device system is presented that is soft and may be worn on the skin [165]. A pressure-responsive element established on membrane deflection is included in the design, as well as a batteryfree, wireless mode of operation that allows for multi-site evaluations around the body. From a pair of main antennas positioned beneath the mattress and coupled to a wireless reader and a multiplexer located at the bedside, such devices provide continuous, simultaneous pressure and temp. evaluations in a sequential readout scheme. Benchtop evaluations and numerical simulations of essential aspects are part of the detection devices and 
system's experimental assessment. Two hemiplegic victims and a tetraplegic victim participated in clinical studies to establish the automation's practicality, usefulness, and longterm stability in operational hospital settings. Figure $12 \mathrm{~b}$ shows the photograph of a device mounted on a body heel that is susceptible to pressure [165].

To address the expectations for future electronic skin application, skin-like sensing devices should be elastic and self-healing. Despite recent significant breakthroughs in skin-inspired electronic materials, imparting these required capabilities to an active semiconductor remains difficult. The combination of a polymer semiconductor and a selfhealing elastomer, both of which are vigorously cross-linked by metal coordination, results in a strain-sensitive, elastic, and autonomously self-healing semiconducting film [166]. The blend film became strain sensitive when the percolation threshold of the polymer semiconductor was controlled, with a gauge factor of $5.75 \times 10^{5}$ at $100 \%$ strain in an elastic transistor. The composite film is also highly elastic and independently self-healable at room temp. The ability to detect strain distribution through surface deformation is proven using a fully integrated $5 \times 5$ elastic active-matrix transistor sensing device array. The photograph of strain-sensitive elastic active-matrix transistor array as a skin-like elastic strain sensing device is shown in figure 12c [166].

Real-time medicare tracking may help forecast and prevent illnesses, as well as enhance treatment by detecting diseases early on. To permit continuous tracking of a person's health, wearable, comfortable sensing devices are necessary; additional significant aspects for this automation are device elasticity, affordable components, and managing, and multifunctionality. To meet these requirements, a flexile, multipurpose printed medicare sensor with a three-axis acceleration sensing device to detect body movement and motion is exhibited [167]. The device features a modular design with two detachable modules, one of which is non-disposable and the other of which is disposable and designed to be worn in contact with the skin. This disposable sensing sheet's design considers hygiene concerns as well as low-cost materials and assembly procedures. It also includes integrated, printed sensing devices to monitor temp., acceleration, and electrocardiograms, as well as a kirigami structure that allows for skin stretching. The device's reusable component incorporates more costly components, as well as a UV light sensing device controlled by carbon nanotube thin-film transistors and a physically flexile and secure liquid metal contact for linking to the disposable sensing sheet. After determining the electrical characteristics of the transistors and flexile sensing devices, a pre-commercial device capable of medicare tracking as well as physical activity detection is proposed, demonstrating that this device is an outstanding podium for the expansion of commercially feasible, wearable medicare monitors. Figure $12 \mathrm{~d}$ (left) shows the image of the multifunctional device attached directly to the skin. Moreover, the real-time acceleration (motion), ECG, skin temp., and UV tracking outcomes are also shown in figure 12d (right) [167]. 


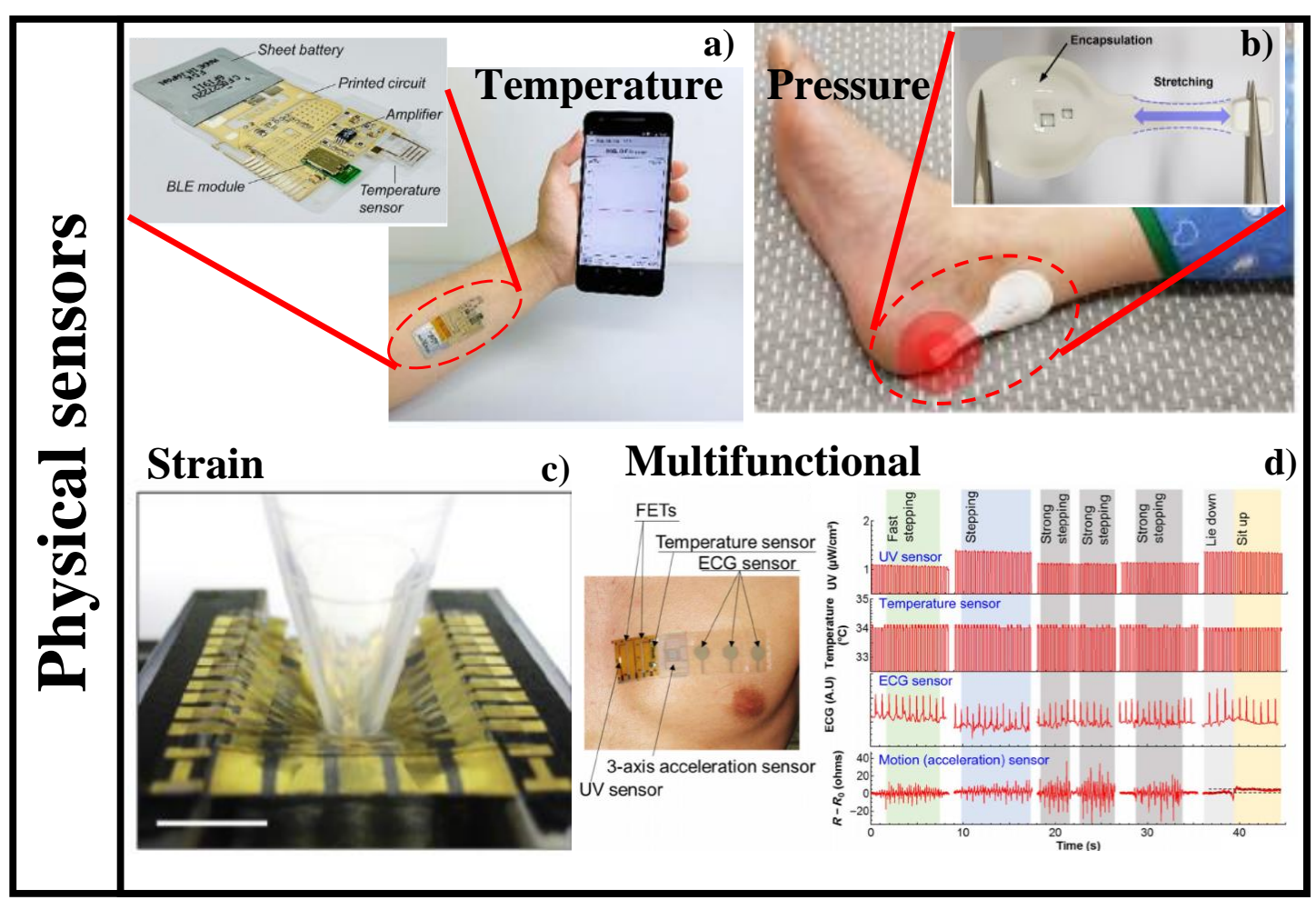

Figure 12. Different applications of flexile physical sensing devices, a) Graphical illustration of wireless sensing platform affixed on an arm for real-time body temp. tracking. Inset shows the optical image of the wireless temp. sensing platform with printed temp. sensing device [161], b) Photograph of a device affixed on body heel that is susceptible to pressure. Inset shows the photograph of a battery-free, wireless pressure sensing device [165], c) Photograph of strain-sensitive elastic active-matrix transistor array as skin-like elastic strain sensing device [166], d) Image of the multifunctional device attached directly onto the skin (left). Real-time acceleration (motion), ECG, skin temp., and UV tracking results [167].

\section{Final remarks}

Life expectancy has risen steadily in most countries during the last several decades, due to considerable advances in medicine, public health, individual and environmental cleanliness. Nevertheless, rising life expectancy combined with dropping birth rates is likely to result in a huge elderly demographic shortly, putting a major strain on these countries' socio-economic structures. As a result, developing cost-effective, easy-to-use solutions for seniors medicare and well-being is critical. Remote health tracking, established on unintrusive and wearable sensors, actuators, and current communication and data technologies, is a cost-efficient option that permits the elderly to remain in their homes rather than expensive medicare institutions. These devices will also permit medicare workers to track key physiological signals of their victims in real-time, analyze health problems, and offer feedback from afar. Owing to unintrusive sampling and high precision, wearable sensors may analyze the abnormal conditions of the physical or chemical components of the body in real-time, exposing the body state in time. Most commercially available wearable devices are mechanically hard modules linked to bands and worn on the wrist, with form factors ultimately limited by the size and weight of the power source batteries. Wearable devices with skin-like characteristics are a relatively new form of automation that is just now making its way out of research laboratories and into pre-commercial prototypes. We presented the recent advances in battery-powered wearable sensors established on optical phenomena, market demand, working mechanism and several commercially available wearable optical sensors. Moreover, skin-like battery-free sensors are also presented in this study, which represents a significant advancement in wearable sensing automation. Several battery-free, wireless OE devices demonstrate the use of near-field communication automation for multicolour light emission and recognition in a way that permits accurate evaluations of the optical characteristics of the skin, to detect PVD and evaluate coloration, and/or of color-responsive materials for environmental detection. Due to their deformability, lightness, mobility, and flexibility, skin-like sensors 
have achieved several functions previously unavailable to traditional sensors. Flexile skinlike sensors may be classified into four types established on the quantities they detect: physical, chemical, physiological, and multifunctional.

Author Contributions: Conceptualization, M.A.B., S.N.K. and N.L.K.; methodology, M.A.B., S.N.K. and N.L.K; software, M.A.B., S.N.K. and N.L.K; validation, M.A.B., S.N.K. and N.L.K; formal analysis, M.A.B., S.N.K. and N.L.K; investigation, M.A.B., S.N.K. and N.L.K; resources, M.A.B., S.N.K. and N.L.K; data curation, M.A.B., S.N.K. and N.L.K; writing-original draft preparation, M.A.B., S.N.K. and N.L.K; writing-review and editing, M.A.B., S.N.K. and N.L.K; visualization, M.A.B., S.N.K. and N.L.K; supervision, M.A.B., S.N.K. and N.L.K; project administration, M.A.B., S.N.K. and N.L.K; funding acquisition, M.A.B., S.N.K. and N.L.K.

Funding: This work was financially supported by the Ministry of Science and Higher Education of the Russian Federation under the Samara National Research University in the overview and comparative parts and under the FSRC "Crystallography and Photonics" of the Russian Academy of Sciences (the state task No. 007-GZ/Ch3363/26) in the part of sensing devices.

Institutional Review Board Statement: No applicable.

Informed Consent Statement: Not applicable.

Acknowledgments: We acknowledge the equal contribution of all the authors.

Conflicts of Interest: The authors declare no conflict of interest.

\section{References}

[1] A. Tricoli, N. Nasiri and S. De, "Wearable and miniturized sensor technologies for personalized and preventive medicine," Advanced functional materials, vol. 27, no. 15, p. 1605271, 2017.

[2] H. Koymedir and A. Ozcan, "Wearable and implantable sensors for biomedical applications," Annu. Rev. Anal. Chem., vol. 11, pp. 127-146, 2018.

[3] Kenry, J. Yeo and C. Lim, "Emerging flexible and wearable physical sensing platforms for healthcare and biomedical applications," Microsystems E Nanoengineering, vol. 2, p. 16043, 2016.

[4] C. Auepanwiriyakul, S. Waibel, J. Songa, P. Bentley and A. Faisal, "Accuracy and acceptability of wearable motion tracking for inpatient monitoring using smartwatches," Sensors, vol. 20, no. 24, p. 7313, 2020.

[5] Y. Fu and J. Liu, "System design for wearable blood oxygen saturation and pulse measurement device," Procedia Manufacturing, vol. 3, pp. 1187-1194, 2015.

[6] E. Casilari, M. Alvarez-Marco and F. Garcia-Lagos, "A study of the use of gyroscope measurements in wearable fall detection systems," Symmetry, vol. 12, p. 649, 2020.

[7] G. B. Lim, "Pacemaker powered by cardiac motion," Nature Reviews Cardiology, vol. 16, p. 386, 2019.

[8] N. Haghjou, M. Soheilian and M. Abdekhodaie, "Sustained release intraocular drug delivery devices for treatment of uveitis," J. Ophthalmic Vis. Res., vol. 6, pp. 317-329, 2011.

[9] R. Lo, P.-Y. Li, S. Saati, R. Agrawal, M. Humayun and E. Meng, "A passive MEMS drug delivery pump for treatment of ocular diseases," Biomed. Microdevices, vol. 11, no. 5, pp. 959-970, 2009.

[10] G. Manivasagam, D. Dhinasekaran and A. Rajamanickam, "Biomedical implants: Corrosion and its prevention- A review," Recent Patents on Corrosion Science, vol. 2, pp. 40-54, 2010.

[11] M. Breteler, E. Huizinga, K. Loon, L. Leenen, D. Dohmen, C. Kalkman and T. Blokhuis, "Reliability of wireless monitoring using a wearable patch sensor in high-risk surgical patients at a step-down unit in the Netherlands: a clinical validation study," BMJ Open, vol. 8, p. e020162, 2018. 
[12] "https://www.sensortips.com/featured/where-are-wearable-sensors-worn/".

[13] M. Moy, S. Mentzer and J. Reilly, "Ambulatory monitoring of cumulative free-living activity," IEEE Eng. Med. Biol. Mag., vol. 22, pp. 89-95, 2003.

[14] D. Sherrill, M. Moy, J. Reilly and P. Bonato, "Using hierarchical clustering methods to classify motor activities of COPD patients from wearable sensor data," J. Neuroeng. Rehabil., vol. 2, p. 16, 2005.

[15] L. Atallah, J. Zhang, B. Lo, D. Shrikrishna, J. Kelly, A. Jackson, M. Polkey, G.-Z. Yang and N. Hopkinson, "Validation of an ear worn sensor for activity monitoring in COPD," Am J. Respir. Crit. Care Med., vol. 181, p. A1211, 2010.

[16] B. Steele, L. Holt, B. Belza, S. Ferris, S. Lakshminaryan and D. Buchner, "Quantitating physical activity in COPD using a triaxial accelerometer," Chest, vol. 117, pp. 1359-1367, 2000.

[17] B. Belza, B. Steele, J. Hunziker, S. Lakshminaryan, L. Holt and D. Buchner, "Correlates of physical activity in chronic obstructive pulmonary disease," Nurs. Res., vol. 50, pp. 195-202, 2001.

[18] A. Hecht, S. Ma, J. Porszasz and R. Casaburi, "Methodology for using long-term accelerometry monitoring to describe daily activity patterns in COPD," COPD, vol. 6, pp. 121-129, 2009.

[19] S. Patel, H. Park, P. Bonato, L. Chan and M. Rodgers, "A review of wearable sensors and systems with application in rehabilitation," Journal of NeuroEngineering and Rehabilitation, vol. 9, p. 21, 2012.

[20] C. Nachiar, N. Ambika, R. Moulika and R. Poovendran, "Design of cost-effective wearable sensors with integrated health monitoring system," in Fourth International Conference on I-SMAC (IoT in Social, Mobile, Analytics and Cloud) (I-SMAC), pp. 12891292, 2020.

[21] G. Cappon, G. Acciaroli, M. Vettoretti, A. Facchinetti and G. Sparacino, "Wearable continuous glucose monitoring sensors: A revolution in diabetes treatment," Electronics, vol. 6, no. 3, p. 65, 2017.

[22] X. Jin, G. Li, T. Xu, L. Su, D. Yan and X. Zhang, "Fully integrated flexible biosensor for wearable continuous glucose monitoring," Biosensors and Bioelectronics, vol. 196, p. 113760, 2022.

[23] M. A. Butt, S. N. Khonina and N. L. Kazanskiy, "Plasmonics: A necessity in the field of sensing-A review (invited)," Fiber and Integrated Optics, p. doi.org/10.1080/01468030.2021.1902590, 2021.

[24] M. A. Butt, S. N. Khonina and N. L. Kazanskiy, "Plasmonic refractive index sensor based on metal-insulator-metal waveguides with high sensitivity," Journal of Modern Optics, vol. 66, no. 9, pp. 1038-1043, 2019.

[25] M. A. Butt, S. N. Khonina and S. N. Kazanskiy, "Silicon on silicon dioxide slot waveguide evanescent field gas absorption sensor," Journal of Modern Optics, vol. 65, no. 2, pp. 174-178, 2018.

[26] M. A. Butt, "Numerical investigation of a small footprint plasmonic Bragg grating structure with a high extinction ratio," Photonics Letters of Poland, vol. 12, no. 3, pp. 82-84, 2020.

[27] M. A. Butt, S. N. Khonina and N. L. Kazanskiy, "Sensitivity enhancement of silicon strip waveguide ring resonator by incorporating a thin metal film," IEEE Sensors Journal, vol. 3, pp. 1355-1362, 2020.

[28] M. A. Butt, S. N. Khonina and N. L. Kazanskiy, "Highly sensitive refractive index sensor based on hybrid plasmonic waveguide microring resonator," Waves in Random and Complex Media, vol. 30, no. 2, pp. 292-299, 2020.

[29] S. N. Khonina, N. L. Kazanskiy and M. A. Butt, "Evanescent field ratio enhancement of a modified ridge waveguide structure for methane gas sensing application," IEEE Sensors Journal, vol. 20, no. 15, pp. 8469-8476, 2020.

[30] M. A. Butt, N. L. Kazanskiy and S. N. Khonina, "Highly sensitive refractive index sensor based on plasmonic Bow Tie configuration," Photonic Sensors, vol. 10, no. 3, pp. 223-232, 2020.

[31] A. Leal-Junior, L. Avellar, A. Frizera and C. Marques, "Smart textiles for multimodal wearable sensing using highly stretchable multiplexed optical fiber system," Scientific Reports, vol. 10, p. 13867, 2020. 
[32] T.-H. Nguyen, L. Mugherli, C. Rivron and T.-H. Tran-Thi, "Innovative colorimetric sensors for the selective detection of monochloramine in air and in water," Sensors and Actuators B: Chemical, vol. 208, pp. 622-627, 2015.

[33] M. Beutler, K. Wiltshire, B. Meyer, C. Moldaenke, C. Luring, M. Meyerhofer, U.-P. Hansen and H. Dau, "A fluorometric method for the differentiation of algal populations in vivo and in situ," Photosynthesis Research, vol. 72, pp. 39-53, 2002.

[34] G. Righini, J. Krzak, A. Lukowiak, G. Macrelli, S. Varas and M. Ferrari, "From flexible electronics to flexible photonics: A brief overview," Optical Materials, vol. 115, p. 111011, 2021.

[35] "Marketresearch.com".

[36] S. Tang, "Wearable sensors for sports performance," in Textiles for Sportswear, 2015, pp. 169-196.

[37] D. Seshadri, R. Li, J. Voos, J. Rowbottom, C. Alfes, C. Zorman and C. Drummond, "Wearable sensors for monitoring the physiological and biochemical profile of the athlete," npj Digital Medicine, vol. 2, p. 72, 2019.

[38] R. Kwasnicki, C.-M. Chen, A. Noakes, S. Hettiaratchy, G.-Z. Yang and A. Darzi, "Developing a wearable sensor for continuous tissue oxygenation monitoring: A proof of concept study," J. Reconstr. Microsurg. Open, vol. 06, no. 01, p. e11, 2021.

[39] S. Majumder, T. Mondal and M. Deen, "A simple, low-cost and efficient gait analyzer for wearable healthcare applications," IEEE Sens. J., vol. 19, pp. 2320-2329, 2019.

[40] L. Johnston, G. Wang, K. Hu, C. Qian and G. Liu, "Advances in biosensors for continuous glucose monitoring towards wearables," Frontiers in Bioengineering and Biotechnology, vol. 9, p. 733810, 2021.

[41] A. Darwish and A. Hassanien, "Wearable and implantable wireless sensor network solutions for healthcare monitoring," Sensors, vol. 11, no. 6, pp. 5561-5595, 2011.

[42] Kong, M; Li, Z; Wu, J; Hu, J; Sheng, Y et al., "A wearable microfluidic device for rapid detection of HIV-1 DNA using recombinase polymerase amplification," Talanta, vol. 205, p. 120155, 2019.

[43] J. Marquard, B. Saver, S. Kandaswamy, V. Martinez, J. Simoni, J. Stekler, D. Ganesan and J. Scanlan, "Designing a wrist-worn sensor to improve medication adherence: accomodating diverse user behaviors and technology preferences," JAMIA Open, vol. 1, no. 2, pp. 153-158, 2018.

[44] R. Varatharajan, G. Manogaran, M. Priyan and R. Sundarasekar, "Wearable sensor devices for early detection of Alzheimer disease using dynamic time warping algorithm," Cluster Computing, vol. 21, pp. 681-690, 2018.

[45] Stavropoulos, T.G et al., "Wearable devices for assessing function in alzheimer's disease: A european public involvement activity about the features and preferences of patients and caregivers," Frontiers in Aging Neuroscience, vol. 13, p. 643135, 2021.

[46] M. Parrilla, T. Guinovart, J. Ferre, P. Blondeau and F. Andrade, "A wearable paper-based sweat sensor for human perspiration monitoring," Adv. Healthc. Mater., vol. 8, p. 1900342, 2019.

[47] G. Cote, R. Lec and M. Pishko, "Emerging biomedical sensing technologies and their applications," IEEE Sens. J., vol. 3, pp. 251-266, 2003.

[48] S. Wang, J. Oh, J. Xu, H. Tran and Z. Bao, "Skin-inspired electronics: An emerging paradigm," Acc. Chem. Res., vol. 51, pp. 1033$1045,2018$.

[49] M. Arif and A. Kattan, "Physical activities monitoring using wearable acceleration sensors attached to the body," PLoS ONE, vol. 10, no. 7, p. e0130851, 2015.

[50] D. Seshadri, R. Li, J. Voos, J. Rowbottom, C. Alfes, C. Zorman and C. Drummond, "Wearable sensors for monitoring the internal and external workload of the athlete," npj Digital Medicine, vol. 2, p. 71, 2019.

[51] J. Dunn, R. Runge and M. Snyder, "Wearables and the medical revolution," Per. Med., vol. 15, no. 5, pp. 429-448, 2018.

[52] Boscari, F; Galasso, S; Acciaroli, G et al., "Head-to-head comparison of the accuracy of Abbott FreeStyle Libre and Dexcom G5 mobile," Nutr. Metab. Cardiovasc. Dis., vol. 28, no. 4, pp. 425-427, 2018. 
[53] Massa, G.G; Gys, I; Op't Eyndt, A et al. , "Evaluation of the Freestyle Libre flash glucose monitoring system in children and adolescents with type 1 diabetes," Horm. Res. Paediatr., vol. 89, no. 3, pp. 189-199, 2018.

[54] Halcox, J.P.J; Wareham, K; Cardew, A et al. , "Assessment of remote heart rhythm sampling using the AliveCor heart monitor to screen for atrial fibrillation: the REHEARSE-AF study," Circulation, vol. 136, no. 19, pp. 1784-1794, 2017.

[55] Tison, G.H; Sanchez, J.M; Ballinger, B et al., "Passive detection of atrial fibrillation using a commercially available smartwatch," JAMA Cardiol., vol. 3, no. 5, pp. 409-416, 2018.

[56] M. Shilaih, V. Clerck, L. Falco, F. Kubler and B. Leeners, "Pulse rate measurement during sleep using wearable sensors, and its correlation with the menstrual cycle phases, a prospective observational study," Sci. Rep., vol. 7, no. 1, p. $1294,2017$.

[57] K. O'Connor, S. Rowson, S. Duma and S. Broglio, "Head-impact-measurement devices: A systematic review," J. Athl. Train., vol. 52, no. 3, pp. 206-227, 2017.

[58] M. Zambotti, L. Rosas, I. Colrain and F. Baker, "The sleep of the ring: Comparison of the OURA sleep tracker against Polysomnography," Behavioral Sleep Medicine, vol. 17, no. 2, pp. 124-136, 2019.

[59] J. Vienneau, J. Bauman, S. Nigg, B. Nigg and S. Jarvis, "Investigating the effects of the SurroGait Rx device on postural stability, gait, and MSIS-29 outcomes in people with multiple sclerosis," Biomed J. Sci. E Tech Res., vol. 2, no. 1, p. $2329,2018$.

[60] Schlachetzki, J.C; Barth, J; Marxreiter, F et al., "Wearable sensors objectively measure gait parameters in Parkinson's disease," PLoS ONE, vol. 12, no. 10, p. e0183989, 2017.

[61] Solomon, M.D; Yang, J; Sung, S.H et al., "Incidence and timing of potentially high-risk arrhythmias detected through long term continuous ambulatory electrocardiographic monitoring," BMC Cardiovasc. Disord., vol. 16, p. $35,2016$.

[62] Sinha, M; Mckeon, K.M; Parker, S et al., "A comparison of time delay in three continuous glucose monitors for adolescents and adults," J. Diabetes Sci. Technol., vol. 11, no. 6, pp. 1132-1137, 2017.

[63] D. Jung, D. Jung and S. Kong, "A lab-on-a-chip-based non-invasive optical sensor for measuring glucose in saliva," Sensors, vol. 17, p. 2607, 2017.

[64] M. Ferrari and V. Quaresima, "A brief review on the history of human functional near-infrared spectroscopy (fNIRS) development and fields of application," Neuroimage, vol. 63, pp. 921-935, 2012.

[65] A. N. Bashkatov, E. Genina, V. I. Kochubey and V. V. Tuchin, "Optical properties of human skin, subcutaneous and mucous tissues in the wavelength range from 400 to 2000 nm," J. Phys. D. Appl. Phys., vol. 38, pp. 2543-2555, 2005.

[66] A. R. Young, "Chromophores in human skin," Phys. Med. Biol., vol. 42, p. 789, 1997.

[67] L. Goldsmith, Physiology, Biochemistry, and Molecular Biology of the Skin, New York, NY, USA: Oxford University Press, 1991.

[68] A. Tarar, U. Mohammad and S. Srivastava, "Wearable skin sensors and their challenges: A review of transdermal, optical, and mechanical sensors," Biosensors, vol. 10, p. 56, 2020.

[69] H. Asada, P. Shaltis, A. Reisner, S. Rhee and R. Hutchinson, "Mobile monitoring with wearable photoplethysmographic biosensors," IEEE Engineering in Medicine, vol. 22, no. 3, pp. 28-40, 2003.

[70] M. Moron, E. Casilari, R. Luque and J. Gazquez, "A wireless monitoring system for pulse-oximetry sensors," in International Conference on Systems Communications, pp.79-84, Montreal, Canada, 2005.

[71] S. Kumar, J. Buckley, J. Barton, M. Pigeon, R. Newberry, M. Rodencal, A. Hajzeraj, T. Hannon, K. Rogers, D. Casey, D. O'Sullivan and B. O'Flynn, "A wristwatch-based wireless sensor platform for IoT health monitoring applications," Sensors, vol. 20 , no. 6 , p. $1675,2020$.

[72] Y.-S. Yan, C. Poon and Y.-T. Zhang, "Reduction of motion artifact in pulse oximetry by smoothed pseudo Wigner-Ville distribution," Journal of NeuroEngineering and Rehabilitation, vol. 2, p. 3, 2005. 
[73] Y. Mendelson and C. Pujary, "Measurement site and photodetector size considerations in optimizing power consumption of a wearable reflectance pulse oximeter," in Proceedings of the 25th Annual International Conference of the IEEE Engineering in Medicine and Biology Society (IEEE Cat. No. 03CH37439), pp. 3016-3019, vol. 4., 2003.

[74] B. Bent, B. Goldstein, W. Kibbe and J. Dunn, "Investigating sources of inaccuracy in wearable optical heart rate sensors," npj Digital Medicine, vol. 3, p. 18, 2020.

[75] Pham, S; Yeap, D et al., "Wearable sensor system to monitor physical activity and the physiological effects of heat exposure," Sensors, vol. 20, no. 3, p. 855, 2020.

[76] G. Millikan, "The oximeter, an instrument for measuring continuously the oxygen saturation of arterial blood in man," Rev. Sci. Instrum., vol. 13, pp. 434-444, 1942.

[77] J. Severinghaus, "Takuo Aoyagi: Discovery of pulse oximetry," Anesth. Analg., vol. 105, pp. S1-S4, 2007.

[78] Bayoumy, K; Gaber, M; Elshafeey, A et al., "Smart wearable devices in cardiovascular care: where we are and how to move forward," Nature Reviews Cardiology, vol. 18, pp. 581-599, 2021.

[79] Y. Mendelson, R. Duckworth and G. Comtois, "A wearable reflectance pulse oximeter for remote physiological monitoring," in 28th Annual International Conference of the IEEE Engineering in Medicine and Biology Society, EMBS '06, pp. 912-915, 2006.

[80] H.-W. Chow and C.-C. Yang, "Accuracy of optical heart rate sensing technology in wearable fitness trackers for young and older adults: validation and comparison study," JMIR Mhealth Uhealth, vol. 8, no. 4, p. e14707, 2020.

[81] P. Colvonen, "Response to: Investigating sources of inaccuracy in wearable optical heart rate sensors," npj Digital Medicine, vol. 4, p. 38, 2021.

[82] E. Guillodo, C. Lemey, M. Simonnet, M. Walter, E. Baca-Garcia, V. Masetti, S. Moga, M. Larsen, H. Network, J. Ropars and S. Berrouiguet, "Clinical applications of mobile health wearable-based sleep monitoring: Systematic review," JMIR Mhealth Uhealth, vol. 8, no. 4, p. e10733, 2020.

[83] A. Ajmal, T. Boonya-Ananta, A. Rodriguez, V. Le and J. Ramella-Roman, "Investigation of optical heart rate sensors in wearables and the influence of skin tone and obesity on photoplethysmography (PPG) signal," in Biophotonics in Exercise Science, Sports Medicine, Health Monitoring Technologies, and Wearables II; 1163808., California, United States, 2021.

[84] J. Smith, E. Bawolek, Y. Lee, B. O'Brien, M. Mans, E. Howard, M. Strnad, J. Christen and M. Goryll, "Application of flexible flat panel display technology to wearable biomedical devices," Electron. Lett., vol. 51, no. 17, pp. 1312-1313, 2015.

[85] S. Pasadyn, M. Soudan, M. Gillinov, P. Houghtaling, D. Phelan, N. Gillinov, B. Bittel and M. Desai, "Accuracy of commercially available heart rate monitors in athletes: a prospective study," Cardiovasc Diagn Ther., vol. 9, no. 4, pp. 379-385, 2019.

[86] J. Choi, R. Ghaffari, L. Baker and J. Rogers, "A skin-interfaced systems for sweat collection and analytics," Sci. Adv., vol. 4, p. eaar3921, 2018.

[87] Heikenfeld, J et al., "Wearable sensors: modalities, challenges, and prospects," Lab Chip, vol. 18, pp. 217-248, 2018.

[88] Y. Haddara, M. Howlader, Y. Haddara and M. Howlader, "Integration of heterogeneous materials for wearable sensors," Polymers, vol. 10, p. 60, 2018.

[89] S. Yao, P. Swetha and Y. Zhu, "Nanomaterial-enabled wearable sensors for healthcare," Healthc. Adv. Healthc. Mater., vol. 7, p. 1700889, 2018.

[90] T. Someya, Z. Bao and G. Malliaras, "The rise of plastic bioelectronics," Nature, vol. 540, pp. 379-385, 2016.

[91] B. Steinberg and J. Piccini, "Screening for atrial fibrillation with a wearable device," JAMA, vol. 320, pp. 139-141, 2018.

[92] Emaminejad, S et al., "Autonomous sweat extraction and analysis applied to cystic fibrosis and glucose monitoring using a fully integrated wearable platform," in Proc. Natl Acad. Sci., USA, 2017.

[93] A. Domschke, "Continuous non-invasive ophthalmic glucose sensor for diabetics," Chimia, vol. 64, pp. 43-44, 2010. 
[94] J. Wang, "Electrochemical glucose biosensors," Chem. Rev., vol. 108, pp. 814-825, 2008.

[95] Rum, L; Sten, O; Vendrame, E et al., "Wearable sensors in sports for persons with disability: A systematic review," Sensors, vol. 21 , no. 5, p. 1858, 2021.

[96] Wang, R; Blackburn, G; Desai, M et al., "Accuracy of Wrist-worn heart rate monitors," JAMA Cardiol., vol. 2, no. 1, pp. 104-106, 2017

[97] S. Gillinov, M. Etiwy, R. Wang, G. Blackburn, D. Phelan, A. Gillinov, P. Houghtaling, H. Javadikasgari and M. Desai, "Variable accuracy of wearable heart rate monitors during aerobic exercise," Med. Sci. Sports Exerc., vol. 49, no. 8, pp. 1697-1703, 2017.

[98] M. Rothmaier, B. Selm, S. Spichtig, D. Haensse and M. Wolf, "Photonic textiles for pulse oximetry," Opt. Express, vol. 16, no. 17, pp. 12973-12986, 2008.

[99] "www.polar.com".

[100] "welcome.moov.cc".

[101] "cosinuss.com".

[102] L. A. DiMeglio, C. Evans-Molina and R. Oram, "Type 1 diabetes," Lancet., vol. 391, pp. 2449-2462, 2018.

[103] A. Olokoba, O. Obateru and L. Olokoba, "Type 2 diabetes mellitus: A review of current trends," Oman Med. J., vol. 27, no. 4, pp. 269-273, 2012.

[104] S. Clarke and J. Foster, "A history of blood glucose meters and their role in self-monitoring of diabetes mellitus," British Journal of biomedical science, vol. 69, no. 2, pp. 83-93, 2012.

[105] I. Hirsch and E. Wright, "Using flash continuous glucose monitoring in primary practice," Clinical diabetes, vol. 37, no. 2, pp. 150-161, 2019.

[106] M. Krakauer, J. Botero, F. Lavalle-Gonzalez, A. Proietti and D. Barbieri, "A review of flash glucose monitoring in type 2 diabetes," Diabetology \& Metabolic Syndrome, vol. 13, p. 42, 2021.

[107] O. Schnell, M. Hanefeld and L. Monnier, "Self-Monitoring of Blood Glucose," J. Diabetes Sci. Technol., vol. 8, no. 3, pp. 609-614, 2014.

[108] J. Kirk and J. Stegner, "Self-monitoring of blood glucose: Practical aspects," J. Diabetes Sci. Technol., vol. 4, no. 2, pp. 435-439, 2010.

[109] H. Lee, Y. Hong, S. Baik, T. Hyeon and D.-H. Kim, "Enzyme-based glucose sensor: From invasive to wearable device," Adv. Healthcare Mater., vol. 7, p. 1701150, 2018.

[110] F. Ribet, G. Stemme and N. Roxhed, "Ultra-miniaturization of a planar amperometric sensor targeting continuous intradermal glucose monitoring," Biosens. Bioelectron., vol. 90, pp. 577-583, 2017.

[111] C. Chen, X. Zhao, Z. Li, Z. Zhu, S. Qian and A. Flewitt, "Current and emerging technology for continuous glucose monitoring," Sensors, vol. 17, p. 182, 2017.

[112] A. Colvin and H. Jiang, "Increased in vivo stability and functional lifetime of an implantable glucose sensor through platinum catalysis," J. Biomed. Mater. Res. Part A, vol. 101, pp. 1274-1282, 2013.

[113] J. Kropff, P. Choudhary, S. Neupane, K. Barnard, S. Bain, C. Kapitza, T. Forst, M. Link, A. Dehennis and J. DeVries, "Accuracy and longevity of an implantable continuous glucose sensor in the PRECISE study: A 180-day, prospective, multicenter, pivotal trial," Diabetes Care, vol. 40, pp. 63-68, 2017.

[114] A. Dehennis, M. Mortellaro and S. Ioacara, "Multisite study of an implanted continuous sensor over 90 days in patients with diabetes mellitus," J. Diabetes Sci. Technol., vol. 9, pp. 951-956, 2015. 
[115] A. Szadkowska, D. Zozilinska-Ziolkiewicz, M. Walczak, K. Cyganek, B. Wolnik, A. Gawrecki and M. Mysliwiec, "Experts opinion: implantable continuous glucose monitoring system-innovation in the management of diabetes," Clinical Diabetology, vol. 8, no. 6, pp. 318-328, 2019.

[116] V. Rachim and W.-Y. Chung, "Wearable-band type visible-near infrared optical biosensor for non-invasive blood glucose monitoring," Sensors and Actuators B: Chemical, vol. 286, pp. 173-180, 2019.

[117] S. Steinhubl, E. Muse and E. Topol, "The emerging field of mobile health," Sci. Transl. Med., vol. 7, p. 283 rv3, 2015.

[118] E. Monton, J. Hernandez, J. Blasco, T. Herve, J. Micallef, I. Grech, A. Brincat and V. Traver, "Body area network for wireless patient monitoring," IET Commun., vol. 2, pp. 215-222, 2008.

[119] X. Liu, Y. Wei and Y. Qiu, "Advanced flexible skin-like pressure and strain sensors for human health monitoring," Micromachines, vol. 12, p. 695, 2021.

[120] B. Ying and X. Liu, "Skin-like hydrogel devices for wearable sensing, soft robotics and beyond," iScience, vol. 24, no. 11, p. 103174, 2021.

[121] Kim, D.-H; Lu, N; Ma, R; Kim, Y.-S; Kim, R.-H; Wang, S; Wu, J; Won, S.M; Tao, H; Islam, A; "Epidermal electronics," Science, vol. 333, pp. 838-843, 2011.

[122] A. Miyamoto, S. Lee, N. Cooray, S. Lee, M. Mori, N. Matsuhisa, H. Jin, L. Yoda, T. Yokota and A. Itoh, "Inflammation-free, gas-permeable, lightweight, stretchable on-skin electronics with nanomeshes," Nat. Nanotechnol., vol. 12, pp. 907-913, 2017.

[123] G. Schwartz, B.-K. Tee, J. Mei, A. Appleton, D. Kim, H. Wang and Z. Bao, "Flexible polymer transistors with high pressure sensitivity for application in electronic skin and health monitoring," Nat. Commun., vol. 4, p. 1859, 2013.

[124] Gao, W; Emaminejad, S; Nyein, H.Y.Y; Challa, S; Chen, K; Peck, A; Fahad, H.M; Ota, H; Shiraki, H; Kiriya, D et al., "Fully integrated wearable sensor arrays for multiplexed in situ perspiration analysis," Nature, vol. 529, pp. 509-514, 2016.

[125] L. Lipani, B. Dupont, F. Doungmene, F. Marken, R. Tyrrell, R. Guy and A. Ilie, "Non-invasive, transdermal, path-selective and specific glucose monitoring via a graphene-based platform," Nat. Nanotechnol., vol. 13, pp. 504-511, 2018.

[126] M. Liu, X. Pu, C. Jiang, T. Liu, X. Huang, L. Chen, C. Du, J. Sun, W. Hu and Z. Wang, "Large-area all-textile pressure sensors for monitoring human motion and physiological signals," Adv. Mater., vol. 29, p. 1703700, 2017.

[127] S. Jung, J. Lee, T. Hyeon, M. Lee and D.-H. Kim, "Fabric-based integrated energy devices for wearable activity monitors," Adv. Mater., vol. 26, pp. 6329-6334, 2014

[128] J. Yang, J. Mun, S. Kwon, S. Park, Z. Bao and S. Park, "Electronic skin: recent progress and future prospects for skin-attachable devices for health monitoring, robotics, and prosthetics," Adv. Mater., p. 1904765, 2019.

[129] C. Ladd, J.-H. So, J. Muth and M. Dickey, "3D printing of free standing liquid metal microstructures," Adv. Mater., vol. 25, pp. 5081-5085, 2013.

[130] Byun, S.-H; Sim, J.Y; Zhou, Z; Lee, J; Qazi, R; Walicki, M.C; Parker, K.E; Haney, M.P; Choi, S.H; Shon, A et al., "Mechanically transformative electronics, sensors, and implantable devices," Sci. Adv., vol. 5, p. eaay0418, 2019.

[131] Liu, Y; Liu, J; Chen, S; Lei, T; Kim, Y; Niu, S; Wang, H; Wang, X et al., "Soft and elastic hydrogel-based microelectronics for localized low-voltage neuromodulation," Nat. Biomed. Eng., vol. 3, pp. 58-68, 2019.

[132] Y. Cao, T. G. Morrissey, E. Acome, S. Allec, B. Wong, C. Keplinger and C. Wang, "A transparent, self-healing, highly stretchable ionic conductor," Adv. Mater., vol. 29, p. 1605099, 2017.

[133] Wang, Y; Zhu, C; Pfattner, R; Yan, H; Jin, L; Chen, S; Molina-Lopez, F; Liu, J; Rabiah, N.I et al., "A highly stretchable, transparent, and conductive polymer," Sci. Adv., vol. 3, p. e1602076, 2017.

[134] H.-J. Kim, K. Sim, A. Thukral and C. Yu, "Rubbery electronics and sensors from intrinsically stretchable elastomeric composites of semiconductors and conductors," Sci. Adv., vol. 3, p. e1701114, 2017. 
[135] Y. Zhao, G. Xing and Z. Chai, "Are carbon nanotubes safe?," Nat. Nanotechnol., vol. 3, pp. 191-192, 2008.

[136] A. D. Maynard, "Are we ready for spray-on carbon nanotubes?," Nat. Nanotechnol., vol. 11, pp. 490-491, 2016.

[137] Z. Zou, C. Zhu, Y. Li, X. Lei, W. Zhang and J. Xiao, "Rehealable, fully recyclable, and malleable electronic skin enabled by dynamic covalent thermoset nanocomposite," Sci. Adv., vol. 4, p. eaaq0508, 2018.

[138] Y. Zhao and X. Huang, "Mechanisms and Materials of flexible and stretchable skin sensors," Micromachines, vol. 8, no. 3, p. 69, 2017.

[139] L. Chen, X. Chen, Z. Zhang, T. Li, T. Zhao, X. Li and J. Zhang, "PDMS-based capacitive pressure sensor for flexible transparent electronics," Journal of Sensors, vol. 2019, p. 1418374, 2019.

[140] D. Lei, N. Liu, T. Su, L. Wang, J. Su, Z. Zhang and Y. Gao, "Research progress of MXenes-based wearable pressure sensors," APL Mater., vol. 8, p. 110702, 2020.

[141] C. Yiu, T. Wong, Y. Liu, K. Yao, L. Zhao, D. Li, Z. Hai, H. Zheng, Z. Wang and X. Yu, "Skin-like strain sensors enabled by elastomer composites for human-machine interfaces," Coatings, vol. 10, no. 8, p. 711, 2020.

[142] C. Wang, T. Yokota and T. Someya , "Natural biopolymer-based biocompatible conductors for stretchable bioelectronics," Chem. Rev., vol. 121, pp. 2109-2146, 2021.

[143] Y. Lei, W. Zhao, Y.-Z. Zhang, Q. Jiang, J. He, A. Baeumner, O. Wolfbeis, Z. Wang, K. Salama and H. Alshareef, "A MXenebased wearable biosensor system for high-performance in vitro perspiration analysis," Small, vol. 15, p. e1901190, 2019.

[144] H. Ota, K. Chen, Y. Lin, D. Kiriya, H. Shiraki, Z. Yu, T.-J. Ha and A. Javey, "Highly deformable liquid-state heterojunction sensors," Nature Communications, vol. 5, p. 5032, 2014.

[145] M. Mannoor, H. Tao, J. Clayton, A. Sengupta, D. Kaplan, R. Naik, N. Verma, F. Omenetto and M. McAlpine, "Graphene-based wireless bacteria detection on tooth enamel," Nat. Commun., vol. 3, p. 763, 2012.

[146] Z. Cao, P. Chen, Z. Ma, S. Li, X. Gao, R.-X. Wu, L. Pan and Y. Shi, "Near-field communication sensors," Sensors, vol. 19, no. 18, p. $3947,2019$.

[147] Reeder, J.T; Choi, J; Xue, Y; Gutruf, P; Hanson, J; Liu, M; Ray, T; Bandodkar, A.J; Avila, R; Xia, W et al., "Waterproof, electronics-enabled, epidermal microfluidic devices for sweat collection, biomarker analysis, and thermography in aquatic settings," Sci. Adv., vol. 5, p. eaau6356, 2019.

[148] Bandodkar, A. J; Gutruf, P; Choi, J; Lee, K; Sekine, Y; Reeder, J.T; Jeang, W.J; Aranyosi, A.J; Lee, S.P; Model, J.B et al., "Batteryfree, skin-interfaced microfluidic/electronic systems for simultaneous electrochemical, colorimetric and volumetric analysis of sweat," Sci. Adv., vol. 5, p. eaav3294, 2019.

[149] S. Aguilar, R. Vidal and C. Gomez, "Opportunistic sensor data collection with bluetooth low energy," Sensors, vol. 17, no. 1, p. 159, 2017.

[150] F. Costa, S. Genovesi, M. Borgese, A. Michel, F. Dicandia and G. Manara, "A review of RFID sensors, the new frontier of internet of things," Sensors, vol. 21, p. 3138, 2021.

[151] S. Niu, N. Matsuhisa, L. Beker, J. Li, S. Wang, J. Wang, Y. Jiang, X. yan, Y. Yun, W. Burnett, A. Poon, J.-H. Tok, X. Chen and Z. Bao, "A wireless body area sensor network based on stretchable passive tags," Nature Electronics, vol. 2, pp. 361-368, 2019.

[152] S.-G. Kang, M.-S. Song, J.-W. Kim, J. Lee and J. Kim, "Near-field communication in biomedical applications," Sensors, vol. 21, no. 3, p. 703, 2021.

[153] Kim, J; Banks, A; Cheng, H et al., "Epidermal electronics with advanced capabilities in near-field communication," Small, vol. 11, no. 8, pp. 906-912, 2015.

[154] F. Alruwaili, K. Cluff, J. Griffith and H. Farhoud, "Passive self resonant skin patch sensor to monitor cardiac intraventricular stroke volume using electromagnetic properties of blood," IEEE J. Transl. Eng. Health Med., vol. 6, p. 1900709, 2018. 
[155] C. Pang, J. Koo, A. Nguyen, J. Caves, M.-G. Kim, A. Chortos, K. Kim, P. Wang, J. Tok and Z. Bao, "Highly skin-conformal microhairy sensor for pulse signal amplification," Advanced Materials, vol. 27, no. 4, pp. 634-640, 2015.

[156] M. Krehel, M. Wolf, L. Boesel, R. Rossi, G.-L. Bona and L. Scherer, "Development of a luminous textile for reflective pulse oximetry measurements," Biomed. Opt. Express, vol. 5, pp. 2537-2547, 2014.

[157] S. Li, Y. Zhang, Y. Wang, K. Xia, Z. Yin, H. Wang, M. Zhang, X. Liang, H. Lu, M. Zhu, H. Wang, X. Shen and Y. Zhang, "Physical sensors for skin-inspired electronics," InfoMat, vol. 2, no. 1, pp. 184-211, 2019.

[158] D. Lipomi, M. Vosgueritchian, B.-K. Tee, S. Hellstrom, J. Lee, C. Fox and Z. Bao, "Skin-like pressure and strain sensors based on transparent elastic films of carbon nanotubes," Nature Nanotechnology, vol. 6, pp. 788-792, 2011.

[159] C. Boutry, Y. Kaizawa, B. Schroeder, A. Chortos, A. Legrand, Z. Wang, J. Chang, P. Fox and Z. Bao, "A stretchable and biodegradable strain and pressure sensor for orthopaedic application," Nat. Electron., vol. 1, pp. 314-321, 2018.

[160] Z. Lei, Q. Wang and P. Wu, "A multifunctional skin-like sensor based on a 3D printed thermo-responsive hydrogel," Materials Horizons, vol. 4, pp. 694-700, 2017.

[161] Y.-F. Wang, T. Sekine, Y. Takeda, K. Yokosawa, H. Matsui, D. Kumaki, T. Shiba, T. Nishikawa and S. Tokito, "Fully printed PEDOT: PSS-based temperature sensor with high humidity stability for wireless healthcare monitoring," Scientific Reports, vol. 10, p. $2467,2020$.

[162] C. Tan, Z. Dong, Y. Li, H. Zhao, X. Huang, Z. Zhou, J.-W. Jiang, Y.-Z. Long, P. Jiang, T.-Y. Zhang and B. Sun, "A high performance wearable strain sensor with advanced thermal management for motion monitoring," Nature Communications, vol. 11, p. 3530, 2020

[163] R. Matsuda, S. Mizuguchi, F. Nakamura, T. Endo, Y. Isoda, G. Inamori and H. Ota, "Highly strechable sensing array for independent detection of pressure and strain exploiting structural and resistive control," Scientific Reports, vol. 10, p. 12666, 2020.

[164] J. Xu, G. Wang, Y. Wu, X. Ren and G. Gao, "Ultrastretchable, wearable strain and pressure sensors based on adhesive, tough, and self-healing hydrogels for human motion monitoring," ACS Appl. Mater. Interfaces, vol. 11, p. 28, 2019.

[165] Oh, Y.S; Kim, J-H; Xie, Z; Cho, S; Han, H et al. , "Battery-free, wireless soft sensors for continuous multi-site measurements of pressure and temperature from patients at risk for pressure injuries," Nature Communications, vol. 12, p. 5008, 2021.

[166] Oh, J.Y; Son, D; Katsumata, T et al. , "Stretchable self-healable semiconducting polymer film for active-matrix strain-sensing array," Materials science, vol. 5, p. eaav3097, 2019.

[167] Y. Yamamoto, S. Harada, D. Yamamoto, W. Honda, T. Arie, S. Akita and K. Takei, "Printed multifunctional flexible device with an integrated motion sensor for health care monitoring," Applied Sciences and Engineering, vol. 2, p. e1601473, 2016. 